Prepared for the U.S. Department of Energy

under Contract DE-AC05-76RL01830

\title{
Hanford Site Environmental Surveillance Master Sampling Schedule for Calendar Year 2010
}

\author{
LE Bisping
}

January 2010

\section{Pacific Northwest}

NATIONAL LABORATORY

Proudly Operated by Battelle Since 1965 


\title{
DISCLAIMER
}

This report was prepared as an account of work sponsored by an agency of the United States Government. Neither the United States Government nor any agency thereof, nor Battelle Memorial Institute, nor any of their employees, makes any warranty, express or implied, or assumes any legal liability or responsibility for the accuracy, completeness, or usefulness of any information, apparatus, product, or process disclosed, or represents that its use would not infringe privately owned rights. Reference herein to any specific commercial product, process, or service by trade name, trademark, manufacturer, or otherwise does not necessarily constitute or imply its endorsement, recommendation, or favoring by the United States Government or any agency thereof, or Battelle Memorial Institute. The views and opinions of authors expressed herein do not necessarily state or reflect those of the United States Government or any agency thereof.

\author{
PACIFIC NORTHWEST NATIONAL LABORATORY \\ operated by \\ BATTELLE \\ for the \\ UNITED STATES DEPARTMENT OF ENERGY \\ under Contract DE-AC05-76RL01830
}

Printed in the United States of America
Available to DOE and DOE contractors from the
Office of Scientific and Technical Information,
P.O. Box 62, Oak Ridge, TN 37831-0062;
ph: (865) 576-8401
fax: $(865) 576-5728$
email: reports@adonis.osti.gov

\begin{abstract}
Available to the public from the National Technical Information Service, U.S. Department of Commerce, 5285 Port Royal Rd., Springfield, VA 22161 ph: (800) 553-6847 fax: $(703) 605-6900$ email: orders@ntis.fedworld.gov online ordering: http://www.ntis.gov/ordering.htm
\end{abstract}

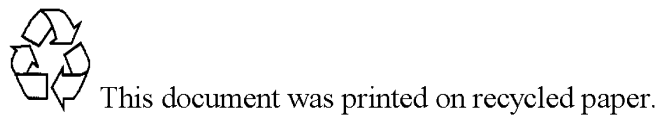




\section{Hanford Site Environmental Surveillance Master Sampling Schedule for Calendar Year 2010}

LE Bisping

January 2010

Prepared for

the U.S. Department of Energy

under Contract DE-AC05-76RL01830

Pacific Northwest National Laboratory

Richland, Washington 99352 


\section{Summary}

Environmental surveillance of the Hanford Site and surrounding areas is conducted by Pacific Northwest National Laboratory ${ }^{1}$ for the U.S. Department of Energy (DOE). Sampling is conducted to evaluate levels of radioactive and nonradioactive pollutants in the Hanford Site environs, as required in DOE Order 450.1A, "Environmental Protection Program,"2 and DOE Order 5400.5, "Radiation Protection of the Public and the Environment." 3 The environmental surveillance sampling design is described in the Hanford Site Environmental Monitoring Plan, United States Department of Energy, Richland Operations Office (DOE/RL-91-50 ${ }^{4}$ ).

This document contains the calendar year 2010 schedule for the routine collection of samples for the Surface Environmental Surveillance Project (SESP) and the Drinking Water Monitoring Project (DWMP). Each section includes sampling locations, sampling frequencies, sample types, and analyses to be performed. In some cases, samples are scheduled on a rotating basis. If a sample will not be collected in 2010, the anticipated year for collection is provided. Maps showing approximate sampling locations are included for media scheduled for collection in 2010.

\section{Surface Environmental Surveillance Project Sampling}

The SESP is a multimedia environmental surveillance effort to measure the concentrations of radionuclides and chemicals in environmental media to demonstrate compliance with applicable environmental quality standards and public exposure limits, and assess environmental impacts. Project personnel annually collect selected samples of ambient air, surface water, agricultural products, fish, wildlife, and sediments. Soil and vegetation samples are collected approximately every 5 years. Analytical capabilities include the measurement of radionuclides at environmental concentrations, and in selected media, nonradiological constituents including metals, anions, volatile organic compounds, and total organic carbon.

\section{Drinking Water Monitoring Project Sampling}

Mission Support Alliance, LLC is responsible for monitoring the quality of drinking water supplied by DOE to its onsite facilities in accordance with federal and state regulations. Pacific Northwest National Laboratory conducts radiological monitoring of onsite drinking water for Mission Support Alliance, LLC concurrent with SESP activities to promote sampling efficiency and consistency, use expertise developed over the years, and reduce costs associated with management, sample collection, procedure development, analytical contracting, data management, quality control, and reporting.

\footnotetext{
${ }^{1}$ Pacific Northwest National Laboratory is operated by Battelle for the U.S. Department of Energy.

2 DOE Order 450.1A. 2008. "Environmental Protection Program.” U.S. Department of Energy, Washington, D.C.

${ }^{3}$ DOE Order 5400.5, Chg 2. 1993. "Radiation Protection of the Public and the Environment.” U.S. Department of Energy, Washington, D.C.

${ }^{4}$ DOE/RL-91-50, Rev. 4. 2008. Environmental Monitoring Plan, United States Department of Energy, Richland Operations Office. U.S. Department of Energy, Richland Operations Office, Richland, Washington.
} 


\section{Data Management}

The Hanford Environmental Information System (HEIS) database is used as a repository for data gathered during environmental surveillance activities at the Hanford Site. For ease in retrieving SESP or drinking water data from the HEIS database, the majority of the location names in this document are the location names used in the database. ${ }^{5}$

\section{Schedule Changes}

This schedule is subject to modification during the year in response to changes in site operations, program requirements, and the nature of the observed results. Operational limitations such as weather, mechanical failures, sample availability, and other factors may also impact scheduled sampling. Therefore, this document may not be an accurate record of samples collected during the year.

\section{Multi-Agency Samples}

By joint agreement, some samples are collected by SESP personnel and provided to the Washington State Department of Health. All planned cooperative sampling efforts are indicated in this schedule.

\section{Additional Information}

Questions relating to the content of this document can be directed to T. M. (Ted) Poston, Manager, SESP, (509) 372-6900 or G. W. (Greg) Patton, Manager, DWMP, (509) 371-7071.

${ }^{5}$ HEIS. 1989. Hanford Environmental Information System. Environmental Database Management, CH2M HILL Plateau Remediation Company, Richland, Washington. 


\section{Acronyms and Symbols}

\section{Acronyms}

$\begin{array}{ll}\text { ALE } & \text { Fitzner/Eberhardt Arid Lands Ecology Reserve } \\ \text { DOE } & \text { U.S. Department of Energy } \\ \text { DOH } & \text { Washington State Department of Health } \\ \text { DR } & \text { Downriver (from noted location) } \\ \text { DWMP } & \text { Drinking Water Monitoring Project } \\ \text { FFTF } & \text { Fast Flux Test Facility } \\ \text { HEIS } & \text { Hanford Environmental Information System } \\ \text { HRM } & \text { Hanford River Marker } \\ \text { ICP-MS } & \text { Inductively coupled plasma mass spectrometry } \\ \text { NASQAN } & \text { National Stream Quality Accounting Network } \\ \text { PNNL } & \text { Pacific Northwest National Laboratory } \\ \text { PRD } & \text { Priest Rivers Dam } \\ \text { SESP } & \text { Surface Environmental Surveillance Project } \\ \text { UR } & \text { Upriver (from noted location) } \\ \text { USGS } & \text { U.S. Geological Survey }\end{array}$

\section{Frequency Symbols Used Symbols}

A

BE

BW

$\mathrm{M}$

M Comp.

$\mathrm{Q}$

Q Comp.

SA

TE annually

biennially (every 2 years)

biweekly (every 2 weeks)

monthly

monthly composite

quarterly

quarterly composite

semiannually (twice each year)

triennially (every 3 years)

\section{Analytical Symbols Used}

Generally, standard element, chemical, and isotope designations are used to indicate the analyses performed. Other analytical designations used include the following:

Alpha

Anions

Beta gross alpha activity of sample

major anions - generally chloride, fluoride, nitrate, nitrite, sulfate

gross beta activity of sample 
Gamma Scan

HTO

Hg-CVAA

Hg-CVAF

ICP-MS

Lo ${ }^{3} \mathrm{H}$

$\mathrm{Pu}$

TOC

$\mathrm{U}$

VOA analysis of photon energy spectrum for individual photon-emitting radionuclides tritiated water $\left({ }^{3} \mathrm{H}^{1} \mathrm{H}^{16} \mathrm{O}\right)$

mercury by cold vapor atomic absorbance spectrometry

total mercury in water by cold vapor atomic fluorescence

major metals by inductively coupled plasma mass spectrometry - samples unfiltered unless otherwise noted

Low-level method for the electrolytic enrichment of tritium

isotopic plutonium $\left({ }^{238} \mathrm{Pu},{ }^{239 / 240} \mathrm{Pu}\right)$

total organic carbon

isotopic uranium $\left({ }^{234} \mathrm{U},{ }^{235} \mathrm{U},{ }^{238} \mathrm{U}\right)$

volatile organic compounds 


\section{Contents}

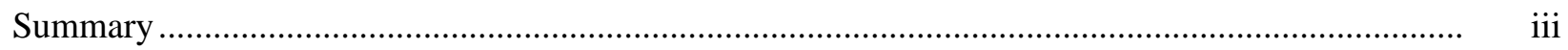

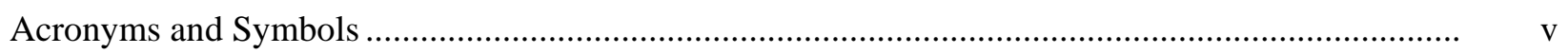

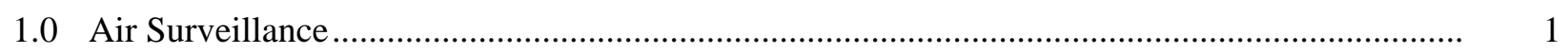

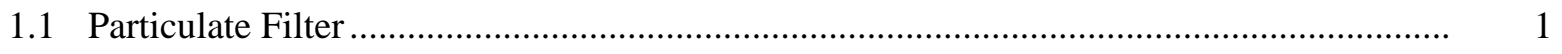

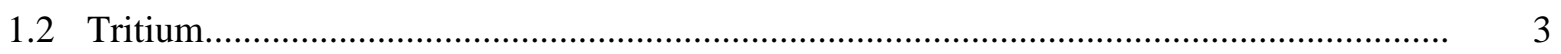

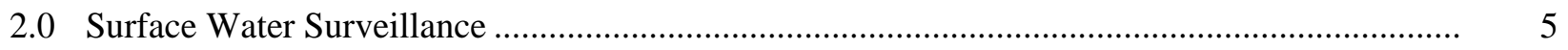

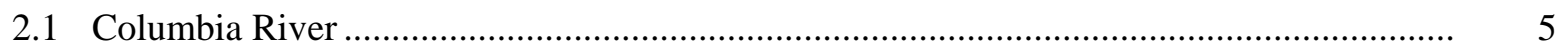

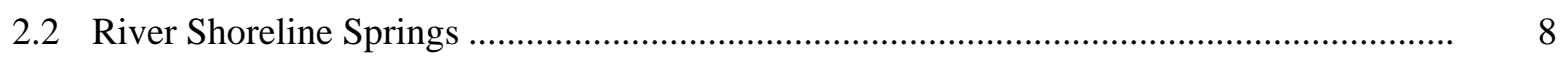

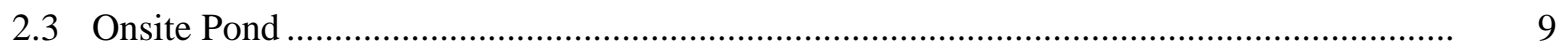

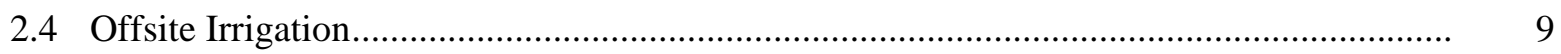

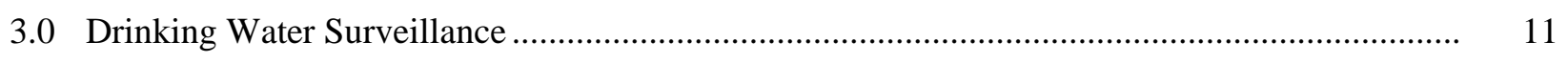

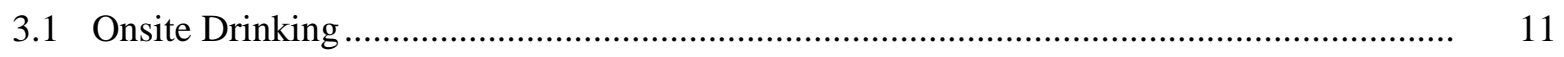

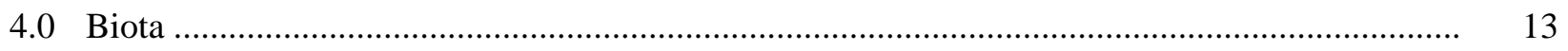

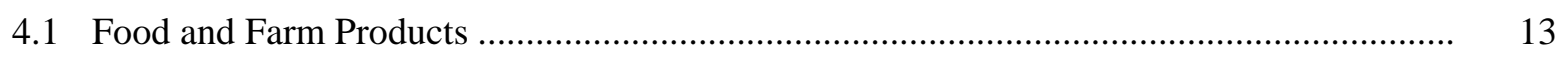

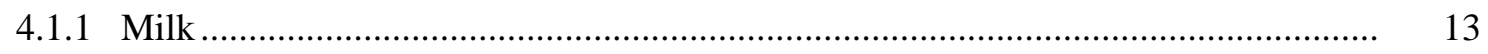

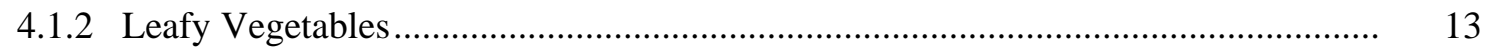

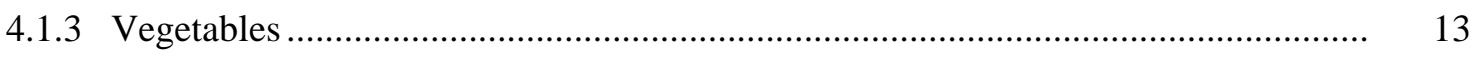

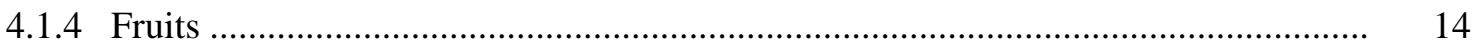

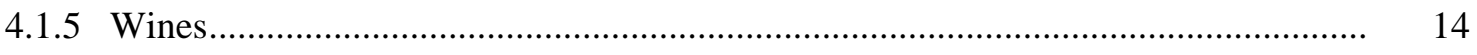

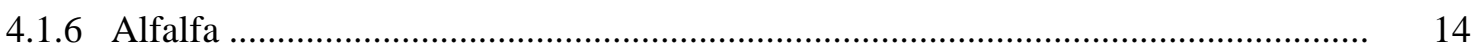

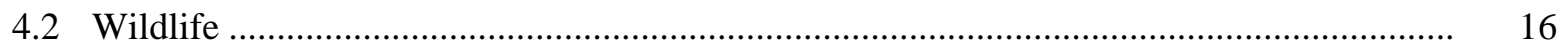

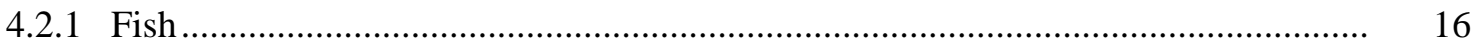

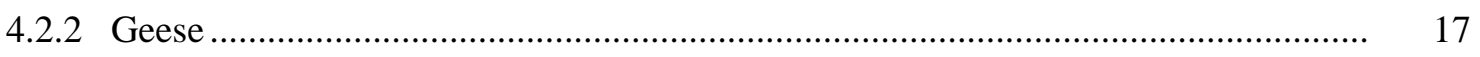

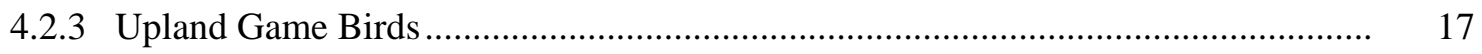

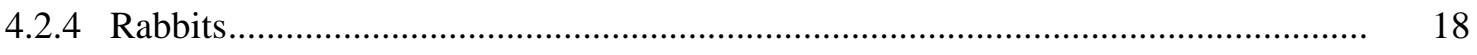

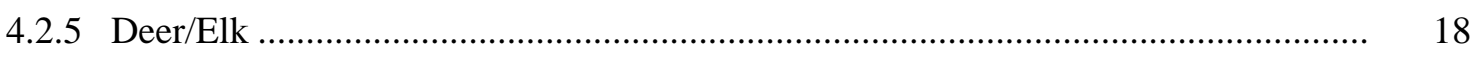

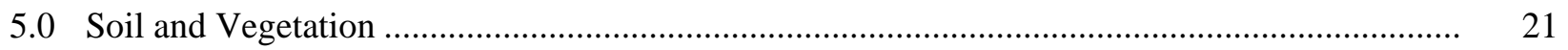

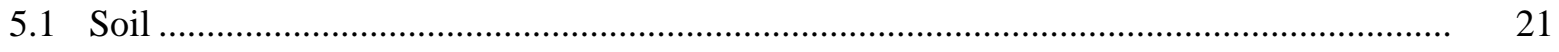

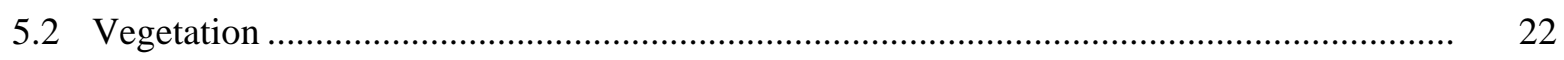

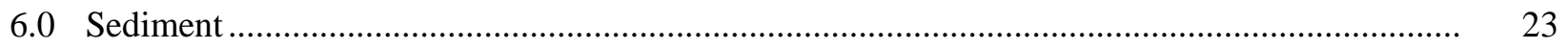

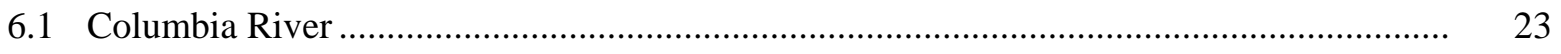

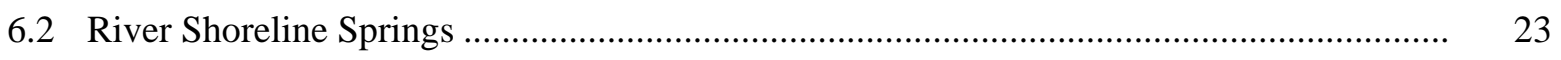

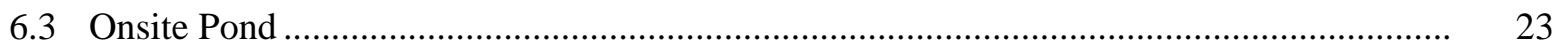




\section{Figures}

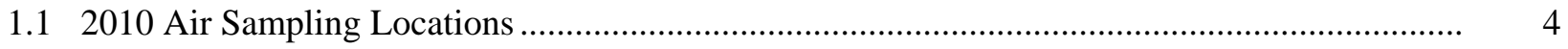

2.12010 Surface Water and Drinking Water Sampling Locations................................................... 10

4.12010 Food and Farm Products Sampling Locations .................................................................. 15

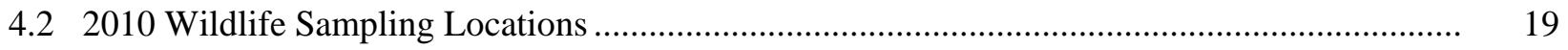

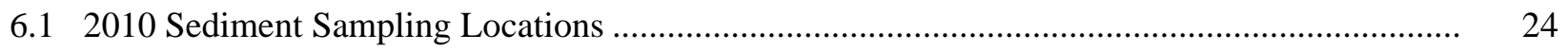




\subsection{Air Surveillance}

\subsection{Particulate Filter}

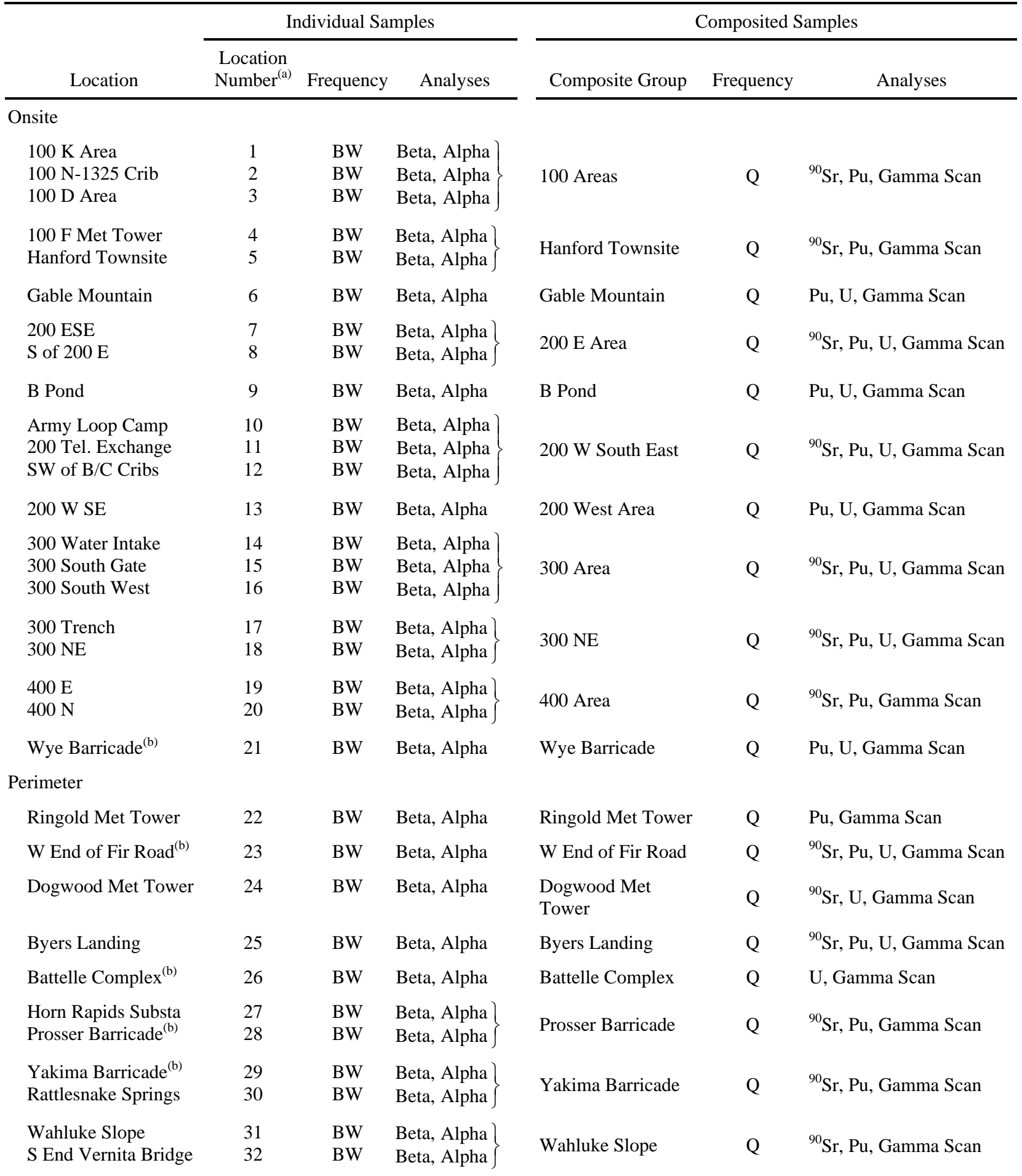




\section{Particulate Filter (contd)}

\begin{tabular}{|c|c|c|c|c|c|c|}
\hline \multirow[b]{2}{*}{ Location } & \multicolumn{3}{|c|}{ Individual Samples } & \multicolumn{3}{|c|}{ Composited Samples } \\
\hline & $\begin{array}{l}\text { Location } \\
\text { Number }^{(a)}\end{array}$ & Frequency & Analyses & Composite Group & Frequency & Analyses \\
\hline \multicolumn{7}{|l|}{ Community } \\
\hline Basin City School & 33 & BW & Beta, Alpha & Basin City School & Q & $\mathrm{Pu}, \mathrm{U}, \mathrm{Gamma}$ Scan \\
\hline Leslie Groves-Rchlnd & 34 & BW & Beta, Alpha & $\begin{array}{l}\text { Leslie Groves- } \\
\text { Rchlnd }\end{array}$ & $\mathrm{Q}$ & ${ }^{90} \mathrm{Sr}, \mathrm{Pu}, \mathrm{U}, \mathrm{Gamma}$ Scan \\
\hline $\begin{array}{l}\text { Pasco } \\
\text { Kennewick-Ely Street }\end{array}$ & $\begin{array}{l}35 \\
36\end{array}$ & $\begin{array}{l}\text { BW } \\
\text { BW }\end{array}$ & $\begin{array}{l}\text { Beta } \\
\text { Beta, Alpha }\end{array}$ & Tri Cities & Q & ${ }^{90} \mathrm{Sr}, \mathrm{Pu}, \mathrm{U}, \mathrm{Gamma}$ Scan \\
\hline Benton City & 37 & BW & Beta & Benton City & $\mathrm{Q}$ & Gamma Scan \\
\hline Mattawa & 38 & BW & Beta & Mattawa & $\mathrm{Q}$ & Gamma Scan \\
\hline Othello & 39 & BW & Beta & Othello & $\mathrm{Q}$ & U, Gamma Scan \\
\hline \multicolumn{7}{|l|}{ Distant } \\
\hline Yakima & 40 & BW & Beta, Alpha & Yakima & Q & ${ }^{90} \mathrm{Sr}, \mathrm{Pu}, \mathrm{U}$, Gamma Scan \\
\hline $\begin{array}{l}\text { (a) Refer to Figure 1.1, } \\
\text { (b) Washington State De }\end{array}$ & $\begin{array}{l}010 \text { Air S } \\
\text { artment of }\end{array}$ & $\begin{array}{l}\text { ampling Loc } \\
\text { Health (DO }\end{array}$ & $\begin{array}{l}\text { cations.” } \\
\text { OH) air samp }\end{array}$ & at this location. & & \\
\hline
\end{tabular}




\subsection{Tritium}

\begin{tabular}{|c|c|c|c|}
\hline Location & $\begin{array}{c}\text { Location } \\
\text { Number }^{(a)}\end{array}$ & Frequency & Analysis $^{(b)}$ \\
\hline \multicolumn{4}{|l|}{ Onsite } \\
\hline $100 \mathrm{~K}$ Area & 1 & M & ${ }^{3} \mathrm{H}$ \\
\hline 100 N-1325 Crib & 2 & M & ${ }^{3} \mathrm{H}$ \\
\hline 200 ESE & 7 & M & ${ }^{3} \mathrm{H}$ \\
\hline 200 Tel. Exchange & 11 & M & ${ }^{3} \mathrm{H}$ \\
\hline 300 Water Intake ${ }^{(\mathrm{c})}$ & 14 & M & ${ }^{3} \mathrm{H}$ \\
\hline 300 South Gate ${ }^{(\mathrm{d})}$ & 15 & M & ${ }^{3} \mathrm{H}$ \\
\hline 300 South West & 16 & M & ${ }^{3} \mathrm{H}$ \\
\hline 300 Trench & 17 & M & ${ }^{3} \mathrm{H}$ \\
\hline $300 \mathrm{NE}$ & 18 & M & ${ }^{3} \mathrm{H}$ \\
\hline $400 \mathrm{E}$ & 19 & M & ${ }^{3} \mathrm{H}$ \\
\hline \multicolumn{4}{|l|}{ Perimeter } \\
\hline Ringold Met Tower & 22 & M & ${ }^{3} \mathrm{H}$ \\
\hline W End of Fir Road & 23 & M & ${ }^{3} \mathrm{H}$ \\
\hline Dogwood Met Tower & 24 & M & ${ }^{3} \mathrm{H}$ \\
\hline Byers Landing & 25 & M & ${ }^{3} \mathrm{H}$ \\
\hline Battelle Complex ${ }^{(c)}$ & 26 & M & ${ }^{3} \mathrm{H}$ \\
\hline Prosser Barricade & 28 & M & ${ }^{3} \mathrm{H}$ \\
\hline Wahluke Slope & 31 & M & ${ }^{3} \mathrm{H}$ \\
\hline \multicolumn{4}{|l|}{ Community } \\
\hline Basin City School & 33 & M & ${ }^{3} \mathrm{H}$ \\
\hline Leslie Groves-Rchlnd & 34 & M & ${ }^{3} \mathrm{H}$ \\
\hline \multicolumn{4}{|l|}{ Distant } \\
\hline Yakima & 40 & M & ${ }^{3} \mathrm{H}$ \\
\hline $\begin{array}{l}\text { (a) Refer to Figure 1.1, } \\
\text { (b) As tritiated water (H } \\
\text { (c) DOH air sampler als } \\
\text { (d) Two tritium samples }\end{array}$ & $\begin{array}{l}\text { location. } \\
\text { ected from }\end{array}$ & ations.” & \\
\hline
\end{tabular}




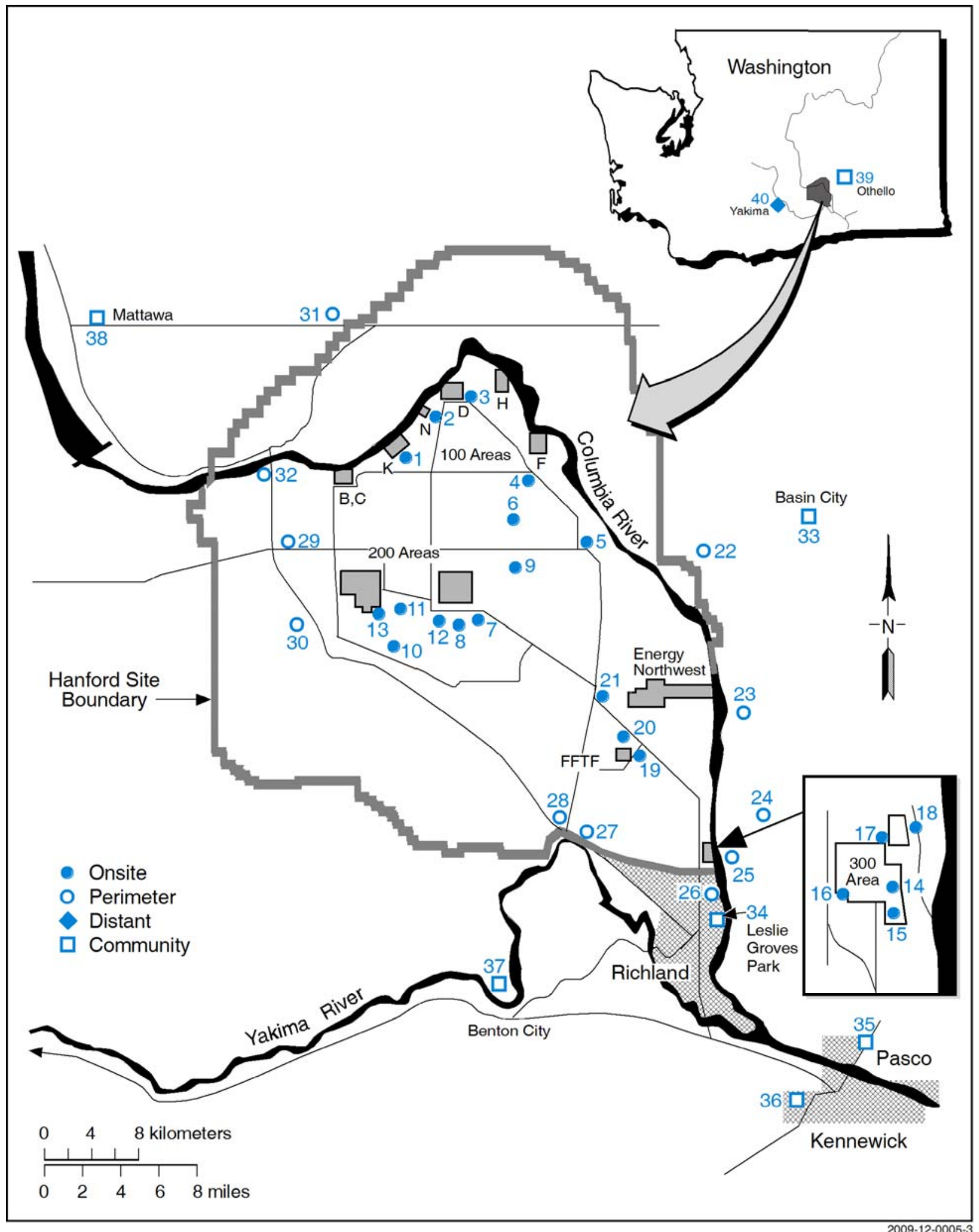

Figure 1.1. 2010 Air Sampling Locations 


\subsection{Surface Water Surveillance}

\subsection{Columbia River}

\begin{tabular}{|c|c|c|c|}
\hline Location $^{(a)}$ & Sample Type & Frequency & Analyses/Agency \\
\hline \multirow[t]{5}{*}{ Priest Rapids-River } & Cumulative & M Comp. ${ }^{(b)}$ & Alpha, Beta, $\mathrm{Lo}^{3} \mathrm{H},{ }^{90} \mathrm{Sr},{ }^{99} \mathrm{Tc}, \mathrm{U} / \mathrm{DOH}^{(\mathrm{c})}$ \\
\hline & Particulate (filter) & M Comp. ${ }^{(d)}$ & Gamma Scan \\
\hline & & Q Comp. ${ }^{(d)}$ & $\mathrm{Pu}$ \\
\hline & Soluble (resin) & M Comp. ${ }^{(d)}$ & Gamma Scan \\
\hline & & Q Comp. ${ }^{(d)}$ & $\mathrm{Pu}$ \\
\hline \multirow[t]{6}{*}{ Rich.Pmphs HRM 46.4} & Cumulative & M Comp..$^{(b)}$ & Alpha, Beta, $\mathrm{Lo}{ }^{3} \mathrm{H},{ }^{90} \mathrm{Sr},{ }^{99} \mathrm{Tc}, \mathrm{U}$ \\
\hline & Particulate (filter) & M Comp. ${ }^{(d)}$ & Gamma Scan \\
\hline & & Q Comp..$^{(\mathrm{d})}$ & $\mathrm{Pu}$ \\
\hline & Soluble (resin) & M Comp. ${ }^{(\mathrm{d})}$ & Gamma Scan \\
\hline & & Q Comp. ${ }^{(\mathrm{d})}$ & $\mathrm{Pu}$ \\
\hline & Grab & $3 / \mathrm{Yr}$ & USGS-NASQAN $^{(\mathrm{e})}$ \\
\hline \multirow[t]{2}{*}{ Rich.Pmphs-1 HRM46.4 ${ }^{(\mathrm{f})}$} & Transect & Q & Lo ${ }^{3} \mathrm{H},{ }^{90} \mathrm{Sr}, \mathrm{U}$, Anions \\
\hline & & A & ICP-MS, Hg-CVAF, ICP-MS Filtered, VOA \\
\hline \multirow[t]{2}{*}{ Rich.Pmphs-2 HRM46.4 } & Transect & Q & Lo ${ }^{3} \mathrm{H},{ }^{90} \mathrm{Sr}, \mathrm{U}$, Anions \\
\hline & & A & ICP-MS, Hg-CVAF, ICP-MS Filtered, VOA \\
\hline \multirow[t]{2}{*}{ Rich.Pmphs-3 HRM46.4 } & Transect & Q & Lo ${ }^{3} \mathrm{H},{ }^{90} \mathrm{Sr}$, U, Anions \\
\hline & & A & ICP-MS, Hg-CVAF, ICP-MS Filtered, VOA \\
\hline \multirow[t]{2}{*}{ Rich.Pmphs-5 HRM46.4 } & Transect & $\mathrm{Q}$ & Lo ${ }^{3} \mathrm{H},{ }^{90} \mathrm{Sr}, \mathrm{U}$, Anions \\
\hline & & A & ICP-MS, Hg-CVAF, ICP-MS Filtered, VOA \\
\hline \multirow[t]{2}{*}{ Rich.Pmphs-7 HRM46.4 } & Transect & $\mathrm{Q}$ & Lo ${ }^{3} \mathrm{H},{ }^{90} \mathrm{Sr}, \mathrm{U}$, Anions \\
\hline & & A & ICP-MS, Hg-CVAF, ICP-MS Filtered, VOA \\
\hline \multirow[t]{2}{*}{ Rich.Pmphs-10 HRM46.4 } & Transect & Q & Lo ${ }^{3} \mathrm{H},{ }^{90} \mathrm{Sr}, \mathrm{U}$, Anions \\
\hline & & A & ICP-MS, Hg-CVAF, ICP-MS Filtered, VOA \\
\hline \multirow[t]{2}{*}{ Rich.Pmphs HRM 43.5} & Transect & $\mathrm{Q}$ & Lo ${ }^{3} \mathrm{H},{ }^{90} \mathrm{Sr}$, U, Anions \\
\hline & & A & ICP-MS, Hg-CVAF, ICP-MS Filtered \\
\hline \multirow[t]{2}{*}{ Rich.Pmphs HRM 43.9} & Transect & Q & Lo ${ }^{3} \mathrm{H},{ }^{90} \mathrm{Sr}, \mathrm{U}$, Anions \\
\hline & & A & ICP-MS, Hg-CVAF, ICP-MS Filtered \\
\hline \multirow[t]{2}{*}{ Rich.Pmphs HRM 45.0} & Transect & $\mathrm{Q}$ & Lo ${ }^{3} \mathrm{H},{ }^{90} \mathrm{Sr}, \mathrm{U}$, Anions \\
\hline & & A & ICP-MS, Hg-CVAF, ICP-MS Filtered \\
\hline \multirow[t]{2}{*}{ Rich.Pmphs HRM 45.8} & Transect & $\mathrm{Q}$ & Lo ${ }^{3} \mathrm{H},{ }^{90} \mathrm{Sr}$, U, Anions \\
\hline & & A & ICP-MS, Hg-CVAF, ICP-MS Filtered \\
\hline Vernita & Grab & 3/Yr & USGS-NASQAN ${ }^{(\mathrm{e})}$ \\
\hline \multirow[t]{2}{*}{ Vernita-1 HRM 0.3} & Transect & $\mathrm{Q}$ & Lo ${ }^{3} \mathrm{H},{ }^{90} \mathrm{Sr}, \mathrm{U}$, Anions \\
\hline & & A & ${ }^{99}$ Tc, ICP-MS, Hg-CVAF, ICP-MS Filtered, VOA \\
\hline \multirow[t]{2}{*}{ Vernita-2 HRM 0.3} & Transect & $\mathrm{Q}$ & Lo ${ }^{3} \mathrm{H},{ }^{90} \mathrm{Sr}$, U, Anions \\
\hline & & A & ${ }^{99}$ Tc, ICP-MS, Hg-CVAF, ICP-MS Filtered, VOA \\
\hline \multirow[t]{2}{*}{ Vernita-3 HRM 0.3} & Transect & Q & Lo ${ }^{3} \mathrm{H},{ }^{90} \mathrm{Sr}, \mathrm{U}$, Anions \\
\hline & & A & ${ }^{99}$ Tc, ICP-MS, Hg-CVAF, ICP-MS Filtered, VOA \\
\hline \multirow[t]{2}{*}{ Vernita-4 HRM 0.3} & Transect & $\mathrm{Q}$ & Lo ${ }^{3} \mathrm{H},{ }^{90} \mathrm{Sr}$, U, Anions \\
\hline & & A & ${ }^{99}$ Tc, ICP-MS, Hg-CVAF, ICP-MS Filtered, VOA \\
\hline
\end{tabular}




\section{Columbia River (contd)}

\begin{tabular}{|c|c|c|c|}
\hline Location $^{(\mathrm{a})}$ & Sample Type & Frequency & Analyses/Agency \\
\hline $100 \mathrm{~N}-1$ HRM $9.5^{(\mathrm{g})}$ & Transect & A & Lo ${ }^{3} \mathrm{H},{ }^{90} \mathrm{Sr}, \mathrm{U}, \mathrm{ICP}-\mathrm{MS}$, ICP-MS Filtered, Anions \\
\hline 100 N -2 HRM 9.5 & Transect & A & Lo ${ }^{3} \mathrm{H},{ }^{90} \mathrm{Sr}, \mathrm{U}$, ICP-MS, ICP-MS Filtered, Anions \\
\hline $100 \mathrm{~N}-3$ HRM 9.5 & Transect & A & Lo ${ }^{3} \mathrm{H},{ }^{90} \mathrm{Sr}, \mathrm{U}$, ICP-MS, ICP-MS Filtered, Anions \\
\hline 100 N -5 HRM 9.5 & Transect & A & Lo ${ }^{3} \mathrm{H},{ }^{90} \mathrm{Sr}$, U, ICP-MS, ICP-MS Filtered, Anions \\
\hline 100 N -7 HRM 9.5 & Transect & A & Lo ${ }^{3} \mathrm{H},{ }^{90} \mathrm{Sr}, \mathrm{U}$, ICP-MS, ICP-MS Filtered, Anions \\
\hline $100 \mathrm{~N}-10$ HRM 9.5 & Transect & A & Lo ${ }^{3} \mathrm{H},{ }^{90} \mathrm{Sr}, \mathrm{U}$, ICP-MS, ICP-MS Filtered, Anions \\
\hline 100 N Shore HRM 8.4 & Transect & A & $\begin{array}{l}\mathrm{Lo}^{3} \mathrm{H},{ }^{90} \mathrm{Sr}, \mathrm{U}, \mathrm{ICP}-\mathrm{MS}, \mathrm{ICP}-\mathrm{MS} \text { Filtered, } \\
\text { Anions/DOH }\end{array}$ \\
\hline 100 N Shore HRM 8.9 & Transect & A & $\begin{array}{l}\text { Lo }{ }^{3} \mathrm{H},{ }^{90} \mathrm{Sr}, \mathrm{U}, \mathrm{ICP}-\mathrm{MS}, \text { ICP-MS Filtered, } \\
\text { Anions/DOH }{ }^{(\mathrm{h})}\end{array}$ \\
\hline 100 N Shore HRM 9.2 & Transect & A & $\begin{array}{l}\text { Lo }{ }^{3} \mathrm{H},{ }^{90} \mathrm{Sr}, \mathrm{U}, \mathrm{ICP}-\mathrm{MS}, \text { ICP-MS Filtered, } \\
\text { Anions } / \mathrm{DOH}^{(\mathrm{h})}\end{array}$ \\
\hline 100 N Shore HRM 9.8 & Transect & A & $\begin{array}{l}\text { Lo }{ }^{3} \mathrm{H},{ }^{90} \mathrm{Sr}, \mathrm{U}, \mathrm{ICP}-\mathrm{MS}, \mathrm{ICP}-\mathrm{MS} \text { Filtered, } \\
\text { Anions/DOH }\end{array}$ \\
\hline Hanfrd TS-1 HRM 28.7 & Transect & A & $\begin{array}{l}\mathrm{Lo}{ }^{3} \mathrm{H},{ }^{90} \mathrm{Sr},{ }^{99} \mathrm{Tc}, \mathrm{U}, \mathrm{ICP}-\mathrm{MS} \text {, ICP-MS Filtered, } \\
\text { Anions }\end{array}$ \\
\hline Hanfrd TS-2 HRM 28.7 & Transect & A & Lo ${ }^{3} \mathrm{H},{ }^{90} \mathrm{Sr}, \mathrm{U}$, ICP-MS, ICP-MS Filtered, Anions \\
\hline Hanfrd TS-3 HRM 28.7 & Transect & A & Lo ${ }^{3} \mathrm{H},{ }^{90} \mathrm{Sr}, \mathrm{U}$, ICP-MS, ICP-MS Filtered, Anions \\
\hline Hanfrd TS-5 HRM 28.7 & Transect & A & Lo ${ }^{3} \mathrm{H},{ }^{90} \mathrm{Sr}, \mathrm{U}$, ICP-MS, ICP-MS Filtered, Anions \\
\hline Hanfrd TS-7 HRM 28.7 & Transect & A & Lo ${ }^{3} \mathrm{H},{ }^{90} \mathrm{Sr}$, U, ICP-MS, ICP-MS Filtered, Anions \\
\hline Hanfrd TS-10 HRM 28.7 & Transect & A & Lo ${ }^{3} \mathrm{H},{ }^{90} \mathrm{Sr}$, U, ICP-MS, ICP-MS Filtered, Anions \\
\hline Hanfrd Twnsite HRM26 & Transect & A & $\begin{array}{l}\text { Lo }{ }^{3} \mathrm{H},{ }^{90} \mathrm{Sr},{ }^{99} \mathrm{Tc}, \mathrm{U}, \mathrm{ICP}-\mathrm{MS} \text {, ICP-MS Filtered, } \\
\text { Anions/DOH }{ }^{\mathrm{h})}\end{array}$ \\
\hline Hanfrd Twnsite HRM27 & Transect & A & $\begin{array}{l}\mathrm{Lo}{ }^{3} \mathrm{H},{ }^{90} \mathrm{Sr},{ }^{99} \mathrm{Tc}, \mathrm{U}, \mathrm{ICP}-\mathrm{MS}, \mathrm{ICP}-\mathrm{MS} \text { Filtered, } \\
\text { Anions } / \mathrm{DOH}^{(\mathrm{h})}\end{array}$ \\
\hline Hanfrd Twnsite HRM28 & Transect & A & $\begin{array}{l}\text { Lo }{ }^{3} \mathrm{H},{ }^{90} \mathrm{Sr},{ }^{99} \mathrm{Tc}, \mathrm{U}, \mathrm{ICP}-\mathrm{MS}, \mathrm{ICP}-\mathrm{MS} \text { Filtered, } \\
\text { Anions/DOH }{ }^{(\mathrm{h})}\end{array}$ \\
\hline Hanfrd Twnsite HRM30 & Transect & A & $\begin{array}{l}\text { Lo }{ }^{3} \mathrm{H},{ }^{90} \mathrm{Sr},{ }^{99} \mathrm{Tc}, \mathrm{U}, \mathrm{ICP}-\mathrm{MS} \text {, ICP-MS Filtered, } \\
\text { Anions/DOH }{ }^{(\mathrm{h})}\end{array}$ \\
\hline 300 Area -1 HRM 43.1 & Transect & A & Lo ${ }^{3} \mathrm{H},{ }^{90} \mathrm{Sr}, \mathrm{U}$, ICP-MS, ICP-MS Filtered, Anions \\
\hline 300 Area -2 HRM 43.1 & Transect & A & Lo ${ }^{3} \mathrm{H},{ }^{90} \mathrm{Sr}, \mathrm{U}$, ICP-MS, ICP-MS Filtered, Anions \\
\hline 300 Area -3 HRM 43.1 & Transect & A & Lo ${ }^{3} \mathrm{H},{ }^{90} \mathrm{Sr}$, U, ICP-MS, ICP-MS Filtered, Anions \\
\hline 300 Area -5 HRM 43.1 & Transect & A & Lo ${ }^{3} \mathrm{H},{ }^{90} \mathrm{Sr}$, U, ICP-MS, ICP-MS Filtered, Anions \\
\hline 300 Area -7 HRM 43.1 & Transect & A & Lo ${ }^{3} \mathrm{H},{ }^{90} \mathrm{Sr}$, U, ICP-MS, ICP-MS Filtered, Anions \\
\hline 300 Area -10 HRM 43.1 & Transect & A & Lo ${ }^{3} \mathrm{H},{ }^{90} \mathrm{Sr}$, U, ICP-MS, ICP-MS Filtered, Anions \\
\hline 300 Area Shr HRM41.5 & Transect & A & $\begin{array}{l}\text { Lo }{ }^{3} \mathrm{H},{ }^{90} \mathrm{Sr}, \mathrm{U}, \mathrm{ICP}-\mathrm{MS}, \text { ICP-MS Filtered, } \\
\text { Anions/DOH }{ }^{(\mathrm{h})}\end{array}$ \\
\hline 300 Area Spring 42-2 & Transect & A & $\begin{array}{l}\text { Lo }{ }^{3} \mathrm{H},{ }^{90} \mathrm{Sr}, \mathrm{U}, \mathrm{ICP}-\mathrm{MS}, \text { ICP-MS Filtered, } \\
\text { Anions/DOH }\end{array}$ \\
\hline 300 Area Spr DR 42-2 & Transect & A & $\begin{array}{l}\mathrm{Lo}^{3} \mathrm{H},{ }^{90} \mathrm{Sr}, \mathrm{U}, \mathrm{ICP}-\mathrm{MS}, \mathrm{ICP}-\mathrm{MS} \text { Filtered, } \\
\text { Anions } / \mathrm{DOH}^{(\mathrm{h})}\end{array}$ \\
\hline 300 Area Shr HRM42.9 & Transect & A & $\begin{array}{l}\text { Lo }{ }^{3} \mathrm{H},{ }^{90} \mathrm{Sr}, \mathrm{U}, \mathrm{ICP}-\mathrm{MS}, \text { ICP-MS Filtered, } \\
\text { Anions/DOH }\end{array}$ \\
\hline
\end{tabular}




\section{Columbia River (contd)}

\begin{tabular}{cccl}
\hline Location $^{(\mathrm{a})}$ & Sample Type & Frequency & \multicolumn{1}{c}{ Analyses/Agency } \\
\hline 300 Area Outfl13 & Grab & $\mathrm{Q}$ & $\mathrm{Lo}^{3} \mathrm{H},{ }^{90} \mathrm{Sr}, \mathrm{U}$, Anions \\
& & $\mathrm{A}$ & ICP-MS, ICP-MS Filtered \\
\hline
\end{tabular}

(a) Refer to Figure 2.1, “2010 Surface Water and Drinking Water Sampling Locations.” Hanford River Markers (HRMs) are a series of signposts along the Hanford Site shoreline of the Columbia River that are roughly $1.6 \mathrm{~km}$ (1 mi) apart. The Vernita Bridge is HRM \#0 and Ferry Street in Richland is HRM \#46. Samples collected between HRMs are assigned a decimal.

(b) Sample is collected weekly and composited monthly for analysis.

(c) Additional sample provided to the DOH (January and June only).

(d) Sample is collected biweekly and composited for analysis.

(e) Analyses are performed by the U.S. Geological Survey (USGS) in conjunction with the National Stream Quality Accounting Network (NASQAN) Program, and include conductance, $\mathrm{pH}$, temperature, turbidity, dissolved oxygen, hardness, Ca, Mg, alkalinity, carbonates, sulfate, Cl, F, solids, $\mathrm{NH}_{4}-\mathrm{N}, \mathrm{NO}_{3}+\mathrm{NO}_{2}, \mathrm{~N}-\mathrm{Kjeldahl,} \mathrm{P}, \mathrm{Cr}$, Fe, and dissolved organic carbon.

(f) Quality assurance sample submitted for analyses twice per year.

(g) Quality assurance sample submitted for analyses once per year.

(h) Additional sample provided to the $\mathrm{DOH}$. 


\subsection{River Shoreline Springs}

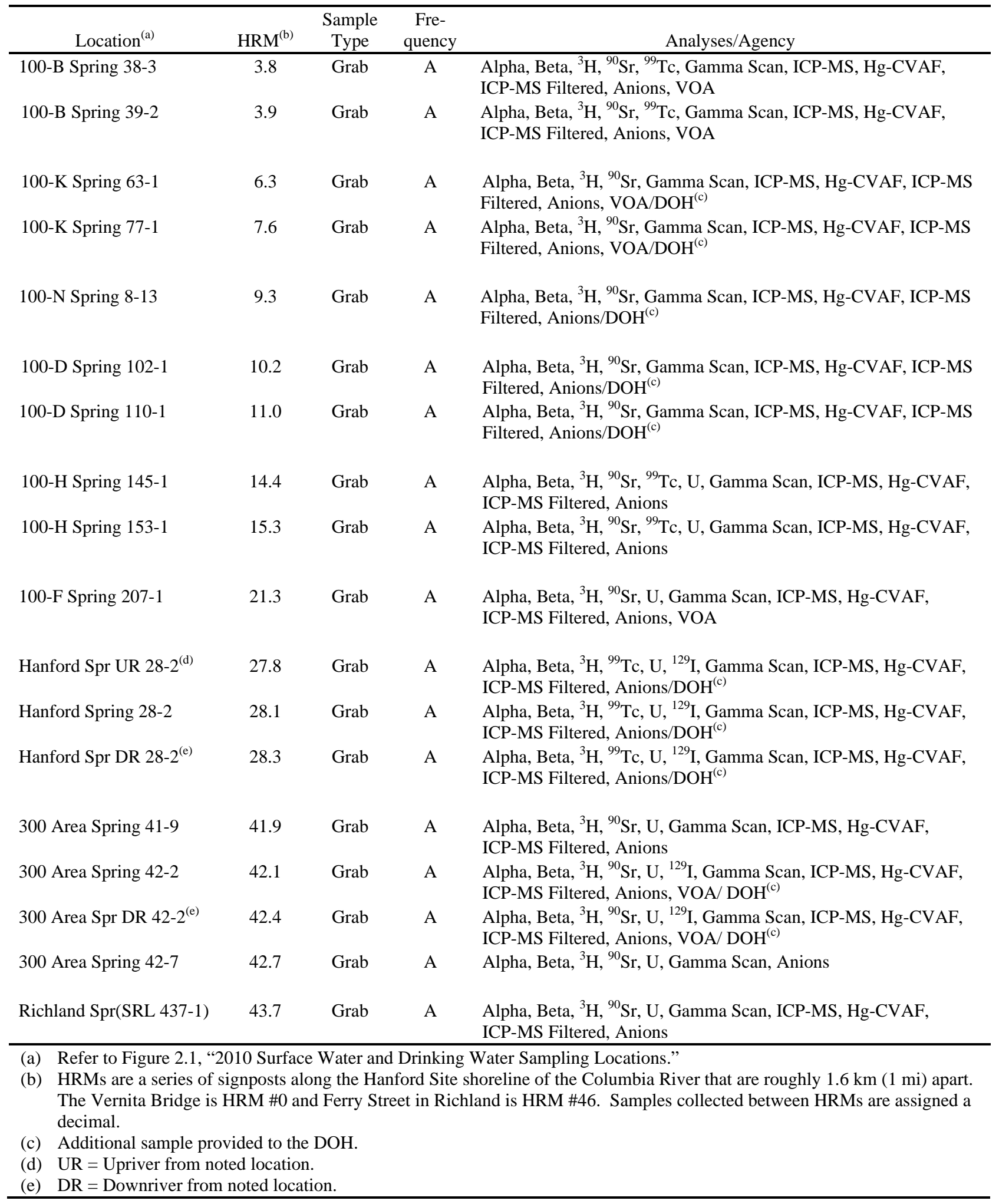




\subsection{Onsite Pond}

\begin{tabular}{|c|c|c|c|}
\hline Location $^{(a)}$ & Sample Type & Frequency & Analyses \\
\hline West Lake & Grab & $\mathrm{Q}$ & ${ }^{3} \mathrm{H}$ \\
\hline FFTF Pond ${ }^{(b)}$ & Grab & $\mathrm{Q}$ & Alpha, Beta, ${ }^{3} \mathrm{H}$, Gamma Scan \\
\hline
\end{tabular}

(a) Refer to Figure 2.1, "2010 Surface Water and Drinking Water Sampling Locations.”

(b) Quality assurance sample submitted for analyses once per year. FFTF = Fast Flux Test Facility.

\subsection{Offsite Irrigation}

\begin{tabular}{lccc}
\hline \multicolumn{1}{c}{ Location $^{(\mathrm{a})}$} & Sample Type & Frequency & Analyses/Agency \\
\hline Riverview Canal & Grab & 3 (May-Sept) & Alpha, Beta, Lo ${ }^{3} \mathrm{H},{ }^{90} \mathrm{Sr}, \mathrm{U}$, Gamma Scan/DOH \\
Horn Rapids Area & Grab & 3 (May-Sept) & Alpha, Beta, Lo ${ }^{3} \mathrm{H},{ }^{90} \mathrm{Sr}, \mathrm{U}, \mathrm{Gamma}$ Scan/DOH ${ }^{(\mathrm{b})}$ \\
\hline
\end{tabular}

(a) Refer to Figure 2.1, "2010 Surface Water and Drinking Water Sampling Locations."

(b) Additional sample provided to the $\mathrm{DOH}$. 


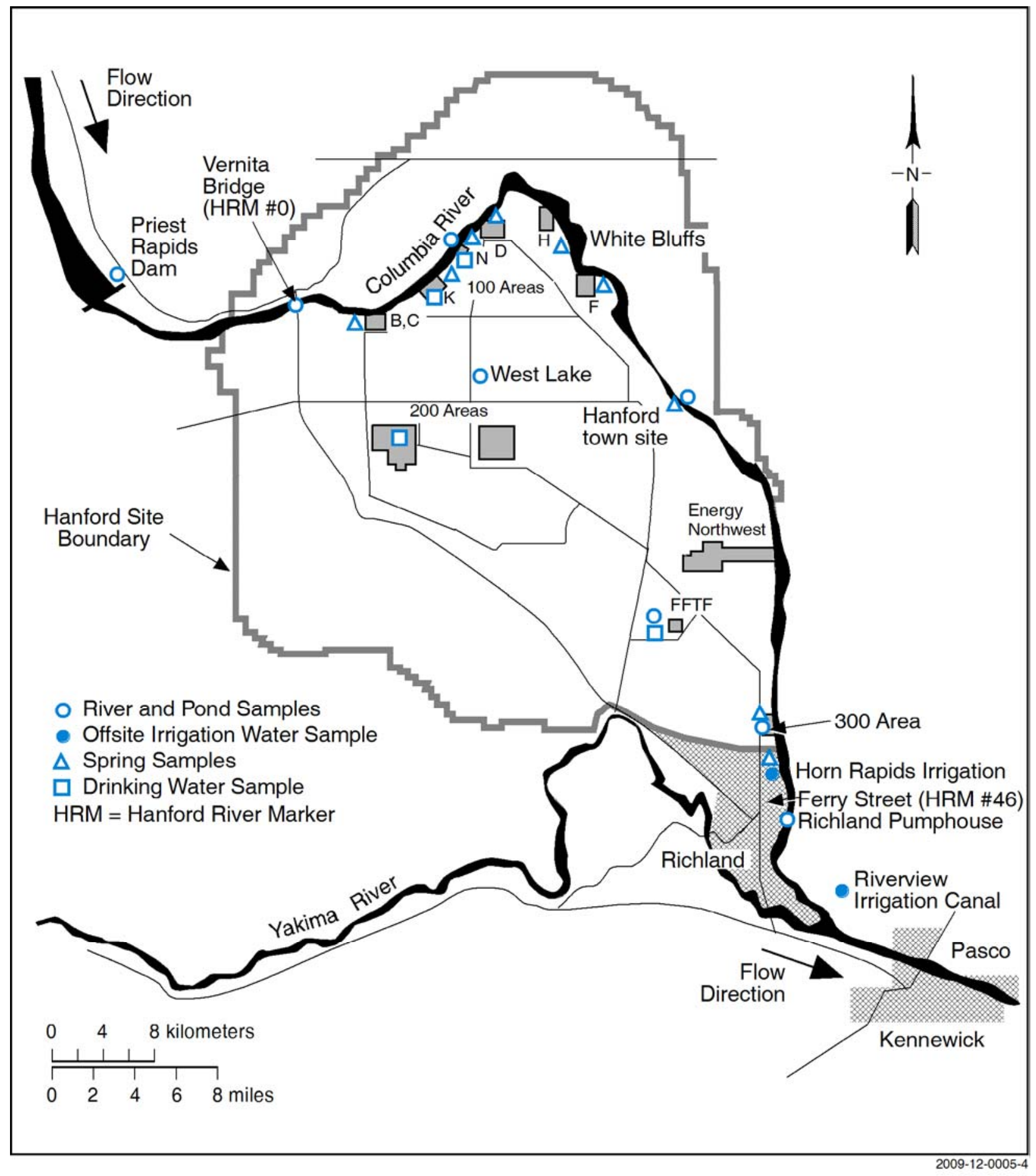

Figure 2.1. 2010 Surface Water and Drinking Water Sampling Locations 


\subsection{Drinking Water Surveillance}

\subsection{Onsite Drinking}

\begin{tabular}{|c|c|c|c|c|c|c|}
\hline \multirow[b]{2}{*}{ Location $^{(\mathrm{a})}$} & \multirow[b]{2}{*}{$\begin{array}{c}\text { Sample } \\
\text { Type }\end{array}$} & \multicolumn{2}{|c|}{ Individual Samples } & \multicolumn{3}{|c|}{ Composited Samples } \\
\hline & & $\begin{array}{c}\text { Fre- } \\
\text { quency }\end{array}$ & Analyses/Agency & Composite Group & $\begin{array}{c}\text { Fre- } \\
\text { quency }\end{array}$ & Analyses/Agency \\
\hline $100 \mathrm{~N}$ Area & Grab & $\mathrm{M}^{(\mathrm{b})}$ & & 100 N Area & $\mathrm{Q}$ & Beta \\
\hline $200 \mathrm{~W}$ Area & Grab & $\mathrm{M}^{(\mathrm{b})}$ & & 200 W Area & $\mathrm{Q}$ & Beta \\
\hline $100 \mathrm{~K}$ Area & Grab & $\mathrm{M}^{(\mathrm{b})}$ & & 100 K Area & Q & Beta \\
\hline 400 Area Well P-14 & Grab & $\mathrm{M}^{(\mathrm{b})}$ & & 400 Area Well P-14 & $\mathrm{Q}$ & Beta \\
\hline 400 Area & Grab & $\mathrm{M}^{(\mathrm{b})}$ & & 400 Area & $\mathrm{Q}$ & Beta \\
\hline $100 \mathrm{~N}$ Area & Grab & $\mathrm{Q}^{(\mathrm{c})}$ & Alpha \} & 100 N Area & A & ${ }^{90} \mathrm{Sr},{ }^{3} \mathrm{H}$ \\
\hline $200 \mathrm{~W}$ Area & Grab & $\mathrm{Q}^{(\mathrm{c})}$ & Alpha \} & 200 W Area & A & ${ }^{90} \mathrm{Sr},{ }^{3} \mathrm{H}$ \\
\hline $100 \mathrm{~K}$ Area & Grab & $\mathrm{Q}^{(\mathrm{c})}$ & Alpha \} & $100 \mathrm{~K}$ Area & A & ${ }^{90} \mathrm{Sr},{ }^{3} \mathrm{H}$ \\
\hline 400 Area Well P-14 & Grab & $\mathrm{Q}^{(\mathrm{c})}$ & Alpha $\}$ & 400 Area Well P-14 & A & ${ }^{90} \mathrm{Sr},{ }^{3} \mathrm{H}$ \\
\hline 400 Area & Grab & $\mathrm{Q}^{(\mathrm{c})}$ & Alpha, $\left.{ }^{3} \mathrm{H} / \mathrm{DOH}^{(\mathrm{d})}\right\}$ & 400 Area & A & ${ }^{90} \mathrm{Sr} / \mathrm{DOH}^{(\mathrm{e})}$ \\
\hline
\end{tabular}

(a) Refer to Figure 2.1, "2010 Surface Water and Drinking Water Sampling Locations.”

(b) Sample is collected monthly and composited for quarterly analysis.

(c) Sample is collected and analyzed quarterly and then composited for annual analysis.

(d) Additional sample provided to the $\mathrm{DOH}$ for ${ }^{3} \mathrm{H}$ analysis (January only).

(e) Additional sample provided quarterly to the $\mathrm{DOH}$ for annual ${ }^{90} \mathrm{Sr}$ analysis. 


\subsection{Biota}

\subsection{Food and Farm Products}

\subsubsection{Milk}

\begin{tabular}{|c|c|c|}
\hline Location $^{(\mathrm{a})}$ & Frequency & Analyses \\
\hline East Wahluke Area $^{(\mathrm{b})}$ & $\mathrm{Q}$ & Lo ${ }^{3} \mathrm{H},{ }^{90} \mathrm{Sr}$, Gamma Scan \\
\hline Sagemoor Composite ${ }^{(\mathrm{b}, \mathrm{c})}$ & Q & Lo ${ }^{3} \mathrm{H},{ }^{90} \mathrm{Sr}$, Gamma Scan \\
\hline Sunnyside Area & $\mathrm{Q}$ & Lo ${ }^{3} \mathrm{H},{ }^{90} \mathrm{Sr}$, Gamma Scan \\
\hline
\end{tabular}

(a) Refer to Figure 4.1, "2010 Food and Farm Products Sampling Locations.”

(b) Sample composited from multiple dairies in each area.

(c) Quality assurance sample submitted for analyses once per year.

\subsubsection{Leafy Vegetables}

\begin{tabular}{|c|c|c|}
\hline Location $^{(\mathrm{a}, \mathrm{b})}$ & Frequency $^{(\mathrm{c})}$ & Analyses/Agency \\
\hline Riverview Area & A & ${ }^{90} \mathrm{Sr}$, Gamma Scan/DOH ${ }^{(\mathrm{d})}$ \\
\hline Sunnyside Area & A & ${ }^{90} \mathrm{Sr}$, Gamma Scan \\
\hline East Wahluke Area & BE (2010) & ${ }^{90} \mathrm{Sr}$, Gamma Scan/DOH ${ }^{(\mathrm{d})}$ \\
\hline Sagemoor Area & BE (2011) & ${ }^{90} \mathrm{Sr}$, Gamma Scan/DOH ${ }^{(\mathrm{d})}$ \\
\hline
\end{tabular}

\footnotetext{
(a) Refer to Figure 4.1, "2010 Food and Farm Products Sampling Locations."

(b) Two samples collected for Pacific Northwest National Laboratory (PNNL) within each area; one sample is analyzed and one is archived.

(c) Samples are collected in 2010 according to their specified frequency unless otherwise noted.

(d) Additional sample provided to the $\mathrm{DOH}$.
}

\subsubsection{Vegetables}

\begin{tabular}{|c|c|c|c|}
\hline Location $^{(\mathrm{a}, \mathrm{b})}$ & Sample Type & Frequency $^{(\mathrm{c})}$ & Analyses/Agency \\
\hline Riverview Area $^{(\mathrm{d})}$ & Potatoes & $\bar{A}$ & ${ }^{90} \mathrm{Sr}$, Gamma Scan/DOH ${ }^{(\mathrm{e})}$ \\
\hline Sunnyside Area & Potatoes & A & ${ }^{90} \mathrm{Sr}$, Gamma Scan \\
\hline East Wahluke Area & Potatoes & A & ${ }^{90} \mathrm{Sr}$, Gamma Scan/DOH ${ }^{(\mathrm{e})}$ \\
\hline Horn Rapids Area & Potatoes & BE (2011) & ${ }^{90} \mathrm{Sr}$, Gamma Scan/DOH ${ }^{(\mathrm{e})}$ \\
\hline Sagemoor Area & Potatoes & TE (2012) & ${ }^{90} \mathrm{Sr}$, Gamma Scan/DOH ${ }^{(\mathrm{e})}$ \\
\hline
\end{tabular}

(a) Refer to Figure 4.1, “2010 Food and Farm Products Sampling Locations.”

(b) Two samples collected for PNNL within each area; one sample is analyzed and one is archived.

(c) Samples are collected in 2010 according to their specified frequency unless otherwise noted.

(d) Other vegetables may be substituted if potatoes are not available.

(e) Additional sample provided to the DOH. 


\subsubsection{Fruits}

\begin{tabular}{|c|c|c|c|c|}
\hline \multirow[b]{2}{*}{ Location $^{(\mathrm{a}, \mathrm{b})}$} & \multicolumn{4}{|c|}{ Collection } \\
\hline & Sample Type & Frequency $^{(\mathrm{c})}$ & Period & Analyses/Agency \\
\hline \multirow[t]{3}{*}{ Sagemoor Area } & Grapes $^{(\mathrm{d})}$ & TE (2010) & September & ${ }^{90} \mathrm{Sr}$, Gamma Scan/DOH ${ }^{(\mathrm{e})}$ \\
\hline & Cherries & TE (2011) & June & ${ }^{90} \mathrm{Sr}$, Gamma Scan/DOH ${ }^{(\mathrm{e})}$ \\
\hline & Apples & TE (2012) & September & ${ }^{90} \mathrm{Sr}$, Gamma Scan/DOH ${ }^{(\mathrm{e})}$ \\
\hline \multirow[t]{4}{*}{ Sunnyside Area } & Grapes $^{(\mathrm{d})}$ & TE (2010) & September & ${ }^{90} \mathrm{Sr}$, Gamma Scan \\
\hline & Tomatoes & $\mathrm{A}$ & July & ${ }^{90} \mathrm{Sr},{ }^{3} \mathrm{H}$, Gamma Scan \\
\hline & Cherries & TE (2011) & June & ${ }^{90} \mathrm{Sr}$, Gamma Scan/DOH ${ }^{(\mathrm{e})}$ \\
\hline & Apples & TE (2012) & September & ${ }^{90} \mathrm{Sr}$, Gamma Scan/DOH ${ }^{(\mathrm{e})}$ \\
\hline \multirow[t]{4}{*}{ Riverview Area $^{(\mathrm{f})}$} & Grapes $^{(d)}$ & TE (2010) & September & ${ }^{90} \mathrm{Sr}$, Gamma Scan/DOH ${ }^{(\mathrm{e})}$ \\
\hline & Tomatoes & A & July & ${ }^{90} \mathrm{Sr},{ }^{3} \mathrm{H}$, Gamma Scan \\
\hline & Cherries & TE (2011) & June & ${ }^{90} \mathrm{Sr}$, Gamma Scan \\
\hline & Apples & TE (2012) & September & ${ }^{90} \mathrm{Sr}$, Gamma Scan/DOH ${ }^{(\mathrm{e})}$ \\
\hline Cold Creek Area & Grapes $^{(\mathrm{d})}$ & TE (2010) & September & ${ }^{90} \mathrm{Sr}$, Gamma Scan \\
\hline Ringold Area & Cherries & TE (2011) & June & ${ }^{90} \mathrm{Sr}$, Gamma Scan/DOH ${ }^{(\mathrm{e})}$ \\
\hline East Wahluke Area & Cherries & TE (2011) & June & ${ }^{90} \mathrm{Sr}$, Gamma Scan/DOH ${ }^{(\mathrm{e})}$ \\
\hline Mattawa Area & Apples & TE (2012) & September & ${ }^{90} \mathrm{Sr}$, Gamma Scan/DOH ${ }^{(\mathrm{e})}$ \\
\hline \multicolumn{5}{|c|}{ (a) Refer to Figure 4.1, “2010 Food and Farm Products Sampling Locations.” } \\
\hline \multicolumn{5}{|c|}{ (b) Two samples collected for the PNNL within each area; one sample is analyzed and one is archived. } \\
\hline \multicolumn{5}{|c|}{ (c) Samples are collected in 2010 according to their specified frequency unless otherwise noted. } \\
\hline \multicolumn{5}{|c|}{ (d) Concord grapes preferred; table grapes acceptable if conc } \\
\hline \multicolumn{5}{|c|}{ (e) Additional sample provided to the $\mathrm{DOH}$. } \\
\hline \multicolumn{5}{|c|}{ (f) Other fruits may be substituted due to availability. } \\
\hline
\end{tabular}

\subsubsection{Wines}

\begin{tabular}{|c|c|c|c|c|}
\hline Location $^{(a)}$ & Sample Type & Frequency & $\begin{array}{c}\text { Collection } \\
\text { Period }\end{array}$ & Analyses/Agency \\
\hline \multirow[t]{2}{*}{ Columbia Basin $^{(\mathrm{b})}$} & White & $\mathrm{BE}(2011)$ & December & $\mathrm{Lo}^{3} \mathrm{H}$, Gamma Scan/DOH ${ }^{(\mathrm{c})}$ \\
\hline & Red & BE (2011) & December & Lo ${ }^{3} \mathrm{H}$, Gamma Scan/DOH ${ }^{(\mathrm{c})}$ \\
\hline \multirow[t]{2}{*}{ Yakima Valley } & White & BE (2011) & December & Lo ${ }^{3} \mathrm{H}$, Gamma Scan/DOH ${ }^{(\mathrm{c})}$ \\
\hline & Red & BE (2011) & December & Lo ${ }^{3} \mathrm{H}$, Gamma Scan/DOH ${ }^{(\mathrm{c})}$ \\
\hline \multirow[t]{2}{*}{ Mattawa Area } & White & BE (2011) & December & $\mathrm{Lo}^{3} \mathrm{H}$, Gamma Scan/DOH ${ }^{(\mathrm{c})}$ \\
\hline & Red & BE (2011) & December & Lo ${ }^{3} \mathrm{H}$, Gamma Scan/DOH ${ }^{(\mathrm{c})}$ \\
\hline $\begin{array}{l}\text { (a) Two samples } \\
\text { (b) Location refer } \\
\text { (c) Additional san }\end{array}$ & $\begin{array}{l}\text { pe collected f } \\
\text { on and Frankl } \\
\text { ided to the Do }\end{array}$ & $\begin{array}{l}\text { within eac } \\
\text { es. }\end{array}$ & & \\
\hline
\end{tabular}

\subsubsection{Alfalfa}

\begin{tabular}{|c|c|c|c|c|}
\hline Location $^{(a)}$ & Sample Type & Frequency & $\begin{array}{l}\text { Collection } \\
\text { Period }\end{array}$ & Analyses/Agency \\
\hline Sagemoor Area & Alfalfa & BE (2011) & May & ${ }^{90} \mathrm{Sr}$, Gamma Scan \\
\hline Riverview Area & Alfalfa & BE (2011) & May & ${ }^{90} \mathrm{Sr}$, Gamma Scan/DOH ${ }^{(\mathrm{b})}$ \\
\hline Sunnyside Area & Alfalfa & BE (2011) & May & ${ }^{90} \mathrm{Sr}$, Gamma Scan \\
\hline Horn Rapids Area & Alfalfa & BE (2011) & May & ${ }^{90} \mathrm{Sr}$, Gamma Scan/DOH ${ }^{(\mathrm{b})}$ \\
\hline
\end{tabular}

(a) Two samples collected for PNNL within each area; one sample is analyzed and one is archived.

(b) Additional sample provided to the $\mathrm{DOH}$. 


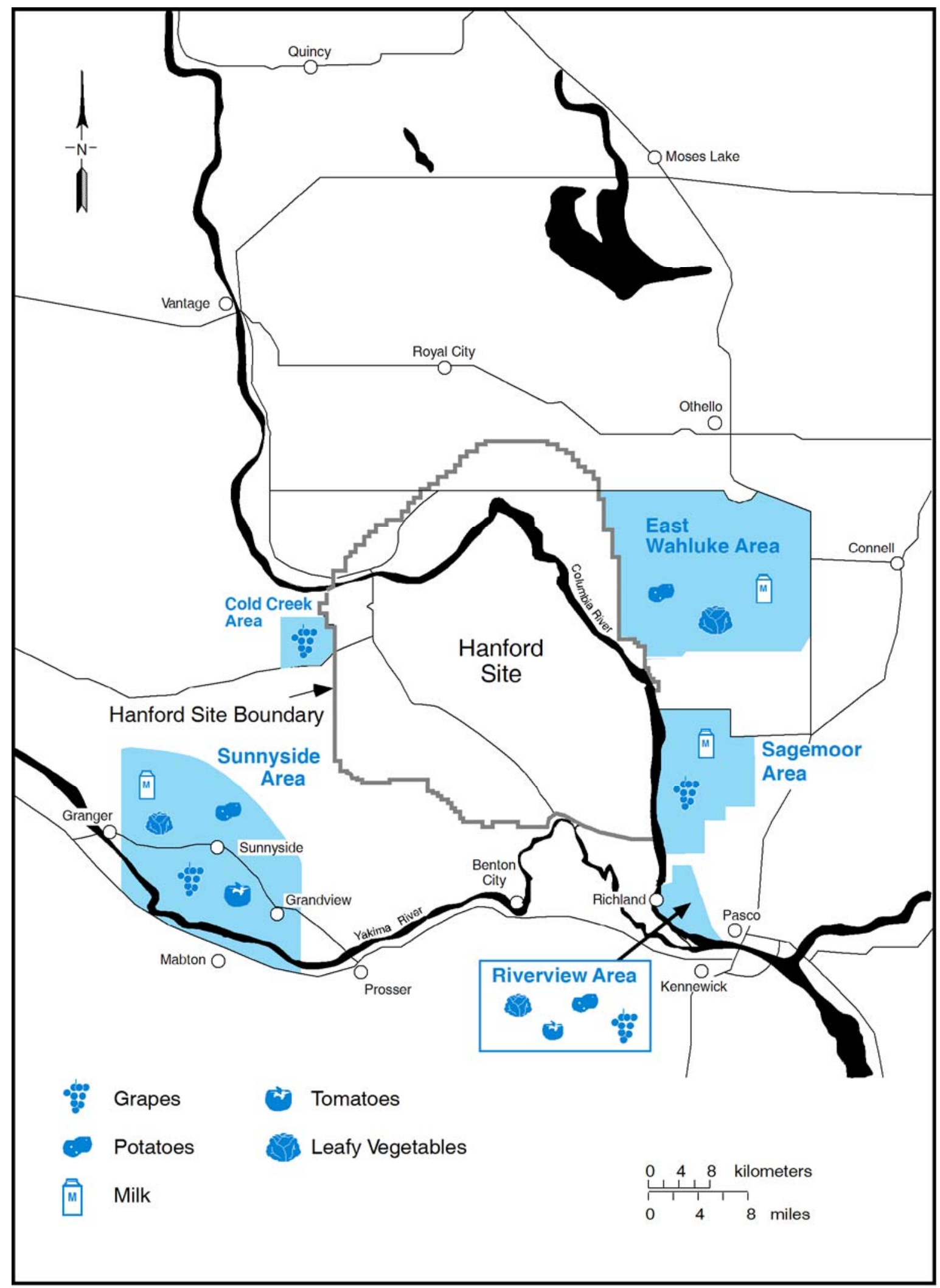

2009-12-0005-2

Figure 4.1. 2010 Food and Farm Products Sampling Locations 


\subsection{Wildlife}

\subsubsection{Fish}

\begin{tabular}{|c|c|c|c|c|c|}
\hline Location $^{(a)}$ & $\begin{array}{c}\text { Species/ } \\
\text { Sample }\end{array}$ & $\begin{array}{c}\text { Number } \\
\text { of Samples }\end{array}$ & Frequency $^{(b)}$ & $\begin{array}{c}\text { Collection } \\
\text { Period }\end{array}$ & Analyses/Agency \\
\hline \multirow[t]{8}{*}{$100-\mathrm{N}$ to $100-\mathrm{D}$} & Carp & & & & \\
\hline & Fillet & 5 & BE (2010) & April-July & Gamma Scan/DOH ${ }^{(\mathrm{c})}$ \\
\hline & Carcass & 5 & BE (2010) & April-July & ${ }^{90} \mathrm{Sr} / \mathrm{DOH}^{(\mathrm{c})}$ \\
\hline & Liver $^{(d)}$ & 5 & BE (2010) & April-July & ICP-MS, Hg-CVAA \\
\hline & Whitefish & & & & \\
\hline & Fillet & 5 & BE (2011) & Oct-Nov & Gamma Scan/DOH ${ }^{(\mathrm{c})}$ \\
\hline & Carcass & 5 & BE (2011) & Oct-Nov & ${ }^{90} \mathrm{Sr} / \mathrm{DOH}^{(\mathrm{c})}$ \\
\hline & Liver $^{(\mathrm{d})}$ & 5 & BE (2011) & Oct-Nov & ICP-MS, Hg-CVAA \\
\hline \multirow[t]{8}{*}{300 Area } & Carp & & & & \\
\hline & Fillet & 5 & BE (2010) & April-July & Gamma Scan, U/DOH ${ }^{(\mathrm{c})}$ \\
\hline & Carcass & 5 & BE (2010) & April-July & ${ }^{90} \mathrm{Sr} / \mathrm{DOH}^{(\mathrm{c})}$ \\
\hline & Liver $^{(\mathrm{d})}$ & 5 & BE (2010) & April-July & ICP-MS, Hg-CVAA \\
\hline & Bass & & & & \\
\hline & Fillet & 5 & TE (2011) & April-June & Gamma Scan, U/DOH ${ }^{(\mathrm{c})}$ \\
\hline & Carcass & 5 & TE (2011) & April-June & ${ }^{90} \mathrm{Sr} / \mathrm{DOH}^{(\mathrm{c})}$ \\
\hline & Liver $^{(\mathrm{d})}$ & 5 & TE (2011) & April-June & ICP-MS, Hg-CVAA \\
\hline \multicolumn{6}{|l|}{ Background - Desert } \\
\hline \multirow[t]{8}{*}{ Aire/Vantage } & Carp & & & & \\
\hline & Fillet & 5 & BE (2010) & Oct-Nov & Gamma Scan, U/DOH ${ }^{(\mathrm{c})}$ \\
\hline & Carcass & 5 & BE (2010) & Oct-Nov & ${ }^{90} \mathrm{Sr} / \mathrm{DOH}^{(\mathrm{c})}$ \\
\hline & Liver $^{(\mathrm{d})}$ & 5 & BE (2010) & Oct-Nov & ICP-MS, Hg-CVAA \\
\hline & Bass & & & & \\
\hline & Fillet & 5 & TE (2011) & April-June & Gamma Scan, U \\
\hline & Carcass & 5 & TE (2011) & April-June & ${ }^{90} \mathrm{Sr}$ \\
\hline & Liver $^{(d)}$ & 5 & TE (2011) & April-June & ICP-MS, Hg-CVAA \\
\hline \multirow[t]{4}{*}{100 F Slough } & Bass & & & & \\
\hline & Fillet & 5 & TE (2011) & April-June & Gamma Scan/DOH ${ }^{(\mathrm{c})}$ \\
\hline & Carcass & 5 & TE (2011) & April-June & ${ }^{90} \mathrm{Sr} / \mathrm{DOH}^{(\mathrm{c})}$ \\
\hline & Liver $^{(d)}$ & 5 & TE (2011) & April-June & ICP-MS, Hg-CVAA \\
\hline \multirow[t]{4}{*}{ Hanford Slough } & Bass & & & & \\
\hline & Fillet & 5 & TE (2011) & April-June & Gamma Scan/DOH ${ }^{(\mathrm{c})}$ \\
\hline & Carcass & 5 & TE (2011) & April-June & ${ }^{90} \mathrm{Sr} / \mathrm{DOH}^{(\mathrm{c})}$ \\
\hline & Liver $^{(d)}$ & 5 & TE (2011) & April-June & ICP-MS, Hg-CVAA \\
\hline \multicolumn{6}{|l|}{ Background - Priest } \\
\hline \multirow[t]{4}{*}{ Rapids/Wanapum Pools } & Whitefish & & & & \\
\hline & Fillet & 5 & BE (2011) & November & Gamma Scan \\
\hline & Carcass & 5 & BE (2011) & November & ${ }^{90} \mathrm{Sr}$ \\
\hline & Liver $^{(\mathrm{d})}$ & 5 & BE (2011) & November & ICP-MS, Hg-CVAA \\
\hline
\end{tabular}

(a) Refer to Figure 4.2, "2010 Wildlife Sampling Locations."

(b) Samples are collected in 2010 according to their specified frequency unless otherwise noted.

(c) Additional whole fish sample provided to the $\mathrm{DOH}$.

(d) Ecological assessment sample. 


\subsubsection{Geese}

\begin{tabular}{|c|c|c|c|c|c|}
\hline Location & $\begin{array}{l}\text { Species/ } \\
\text { Sample }\end{array}$ & $\begin{array}{c}\text { Number } \\
\text { of Samples }\end{array}$ & Frequency & $\begin{array}{l}\text { Collection } \\
\text { Period }\end{array}$ & Analyses \\
\hline \multirow[t]{4}{*}{100 Areas } & Canada Goose & & & & \\
\hline & Muscle & 5 & BE (2011) & May-July & Gamma Scan \\
\hline & Bone & 5 & BE (2011) & May-July & ${ }^{90} \mathrm{Sr}$ \\
\hline & Liver $^{(a)}$ & 5 & BE (2011) & May-July & ICP-MS, Hg-CVAA \\
\hline \multirow{5}{*}{$\begin{array}{l}\text { Hanf Townsite to } \\
300 \text { Area }\end{array}$} & & & & & \\
\hline & Canada Goose & & & & \\
\hline & Muscle & 5 & BE (2011) & May-July & Gamma Scan \\
\hline & Bone & 5 & BE (2011) & May-July & ${ }^{90} \mathrm{Sr}$ \\
\hline & Liver $^{(a)}$ & 5 & BE (2011) & May-July & ICP-MS, Hg-CVAA \\
\hline \multirow{5}{*}{$\begin{array}{l}\text { Background - } \\
\text { Desert Aire/Vantage }\end{array}$} & & & & & \\
\hline & Canada Goose & & & & \\
\hline & Muscle & 5 & BE (2011) & May-July & Gamma Scan \\
\hline & Bone & 5 & BE (2011) & May-July & ${ }^{90} \mathrm{Sr}$ \\
\hline & Liver $^{(a)}$ & 5 & BE (2011) & May-July & ICP-MS, Hg-CVAA \\
\hline
\end{tabular}

\subsubsection{Upland Game Birds}

\begin{tabular}{|c|c|c|c|c|c|}
\hline Location $^{(a)}$ & $\begin{array}{l}\text { Species/ } \\
\text { Sample }^{(\mathrm{b})}\end{array}$ & $\begin{array}{c}\text { Number } \\
\text { of Samples }\end{array}$ & Frequency & $\begin{array}{c}\text { Collection } \\
\text { Period }\end{array}$ & Analyses/Agency \\
\hline \multirow[t]{4}{*}{$100-\mathrm{D}$ to $100-\mathrm{H}$} & Pheasant & & & & \\
\hline & Muscle & 4 & BE (2010) & September & Gamma Scan \\
\hline & Bone & 4 & BE (2010) & September & ${ }^{90} \mathrm{Sr}$ \\
\hline & Liver $^{(\mathrm{c})}$ & 4 & BE (2010) & September & ICP-MS \\
\hline \multirow[t]{4}{*}{$100-\mathrm{H}$ to $100-\mathrm{F}$} & Pheasant & & & & \\
\hline & Muscle & 6 & BE (2010) & September & Gamma Scan/DOH ${ }^{(\mathrm{d})}$ \\
\hline & Bone & 6 & BE (2010) & September & ${ }^{90} \mathrm{Sr} / \mathrm{DOH}^{(\mathrm{d})}$ \\
\hline & Liver $^{(\mathrm{c})}$ & 6 & BE (2010) & September & ICP-MS \\
\hline \multirow[t]{4}{*}{ Background } & Pheasant & & & & \\
\hline & Muscle & 5 & BE (2010) & September & Gamma Scan/DOH ${ }^{(\mathrm{d})}$ \\
\hline & Bone & 5 & BE (2010) & September & ${ }^{90} \mathrm{Sr} / \mathrm{DOH}^{(\mathrm{d})}$ \\
\hline & Liver $^{(\mathrm{c})}$ & 5 & BE (2010) & September & ICP-MS \\
\hline \multicolumn{6}{|c|}{ Refer to Figure 4.2, “2010 Wildlife Sampling Locations.” } \\
\hline \multicolumn{6}{|c|}{ Pheasants preferred; chukars or quail acceptable if pheasants are unavailable. } \\
\hline \multicolumn{6}{|c|}{ Ecological assessment sample. } \\
\hline \multicolumn{6}{|c|}{ Additional whole bird sample provided to the $\mathrm{DOH}$. } \\
\hline
\end{tabular}




\subsubsection{Rabbits}

\begin{tabular}{|c|c|c|c|c|c|}
\hline Location & $\begin{array}{l}\text { Species/ } \\
\text { Sample }\end{array}$ & $\begin{array}{c}\text { Number } \\
\text { of Samples }\end{array}$ & Frequency & $\begin{array}{c}\text { Collection } \\
\text { Period }\end{array}$ & Analyses/Agency \\
\hline \multirow[t]{4}{*}{$100 \mathrm{~N}$ Area } & Cottontail & & & & \\
\hline & Muscle & 4 & BE (2011) & Jan-Dec & Gamma Scan/DOH ${ }^{(a)}$ \\
\hline & Bone & 4 & BE (2011) & Jan-Dec & ${ }^{90} \mathrm{Sr} / \mathrm{DOH}^{(\mathrm{a})}$ \\
\hline & Liver $^{(b)}$ & 4 & BE (2011) & Jan-Dec & ICP-MS \\
\hline \multirow[t]{4}{*}{200 E Area } & Cottontail & & & & \\
\hline & Muscle & 4 & BE (2011) & Jan-Dec & Gamma Scan/DOH ${ }^{(a)}$ \\
\hline & Bone & 4 & BE (2011) & Jan-Dec & ${ }^{90} \mathrm{Sr} / \mathrm{DOH}^{(\mathrm{a})}$ \\
\hline & Liver $^{(b)}$ & 4 & BE (2011) & Jan-Dec & $\mathrm{Pu}, \mathrm{ICP}-\mathrm{MS}$ \\
\hline \multirow[t]{4}{*}{200 West } & Cottontail & & & & \\
\hline & Muscle & 4 & BE (2011) & Jan-Dec & Gamma Scan/DOH ${ }^{(\mathrm{a})}$ \\
\hline & Bone & 4 & BE (2011) & Jan-Dec & ${ }^{90} \mathrm{Sr} / \mathrm{DOH}^{(\mathrm{a})}$ \\
\hline & Liver $^{(b)}$ & 4 & BE (2011) & Jan-Dec & Pu, ICP-MS \\
\hline \multirow[t]{4}{*}{ Background } & Cottontail & & & & \\
\hline & Muscle & 5 & BE (2011) & Jan-Dec & Gamma Scan \\
\hline & Bone & 5 & BE (2011) & Jan-Dec & ${ }^{90} \mathrm{Sr}$ \\
\hline & Liver $^{(b)}$ & 5 & BE (2011) & Jan-Dec & $\mathrm{Pu}, \mathrm{ICP}-\mathrm{MS}$ \\
\hline
\end{tabular}

(a) Additional whole rabbit sample provided to the $\mathrm{DOH}$.

(b) Ecological assessment sample.

\subsubsection{Deer/Elk}

\begin{tabular}{|c|c|c|c|c|c|}
\hline Location $^{(a)}$ & $\begin{array}{l}\text { Species/ } \\
\text { Sample }\end{array}$ & $\begin{array}{c}\text { Number } \\
\text { of Samples }\end{array}$ & Frequency & $\begin{array}{l}\text { Collection } \\
\text { Period }\end{array}$ & Analyses/Agency \\
\hline \multirow[t]{4}{*}{$100 \mathrm{~N}$ Area } & Mule Deer & & & & \\
\hline & Muscle & 2 & BE (2010) & Nov-Dec & Gamma Scan/DOH ${ }^{(b)}$ \\
\hline & Bone & 2 & BE (2010) & Nov-Dec & ${ }^{90} \mathrm{Sr} / \mathrm{DOH}^{(\mathrm{b})}$ \\
\hline & Liver $^{(\mathrm{c})}$ & 2 & BE (2010) & Nov-Dec & ICP-MS \\
\hline \multirow[t]{4}{*}{200 Areas } & Mule Deer & & & & \\
\hline & Muscle & 2 & $\mathrm{BE}(2010)$ & Nov-Dec & Gamma Scan/DOH ${ }^{(b)}$ \\
\hline & Bone & 2 & BE (2010) & Nov-Dec & ${ }^{90} \mathrm{Sr} / \mathrm{DOH}^{(\mathrm{b})}$ \\
\hline & Liver $^{(\mathrm{c})}$ & 2 & BE (2010) & Nov-Dec & $\mathrm{Pu}, \mathrm{ICP}-\mathrm{MS}$ \\
\hline \multirow{4}{*}{$\begin{array}{l}\text { Road Kill at Onsite } \\
\text { Locations }^{(\mathrm{d})}\end{array}$} & & & & & \\
\hline & Mule Deer or Elk & & & & \\
\hline & Muscle & 10 & BE (2010) & As Available & Gamma Scan \\
\hline & Bone & 10 & BE (2010) & As Available & ${ }^{90} \mathrm{Sr}$ \\
\hline \multirow[t]{4}{*}{ Background $^{(\mathrm{e})}$} & Mule Deer & & & & \\
\hline & Muscle & 2 & BE (2010) & October & Gamma Scan/DOH ${ }^{(b)}$ \\
\hline & Bone & 2 & BE (2010) & October & ${ }^{90} \mathrm{Sr} / \mathrm{DOH}^{(\mathrm{b})}$ \\
\hline & Liver $^{(\mathrm{c})}$ & 2 & BE (2010) & October & $\mathrm{Pu}, \mathrm{ICP}-\mathrm{MS}$ \\
\hline
\end{tabular}

(a) Refer to Figure 4.2, "2019Wildlife Sampling Locations.”

(b) Additional sample provided to the $\mathrm{DOH}$.

(c) Ecological assessment sample.

(d) As available, according to location.

(e) One of the two background samples obtained from the DOH. 


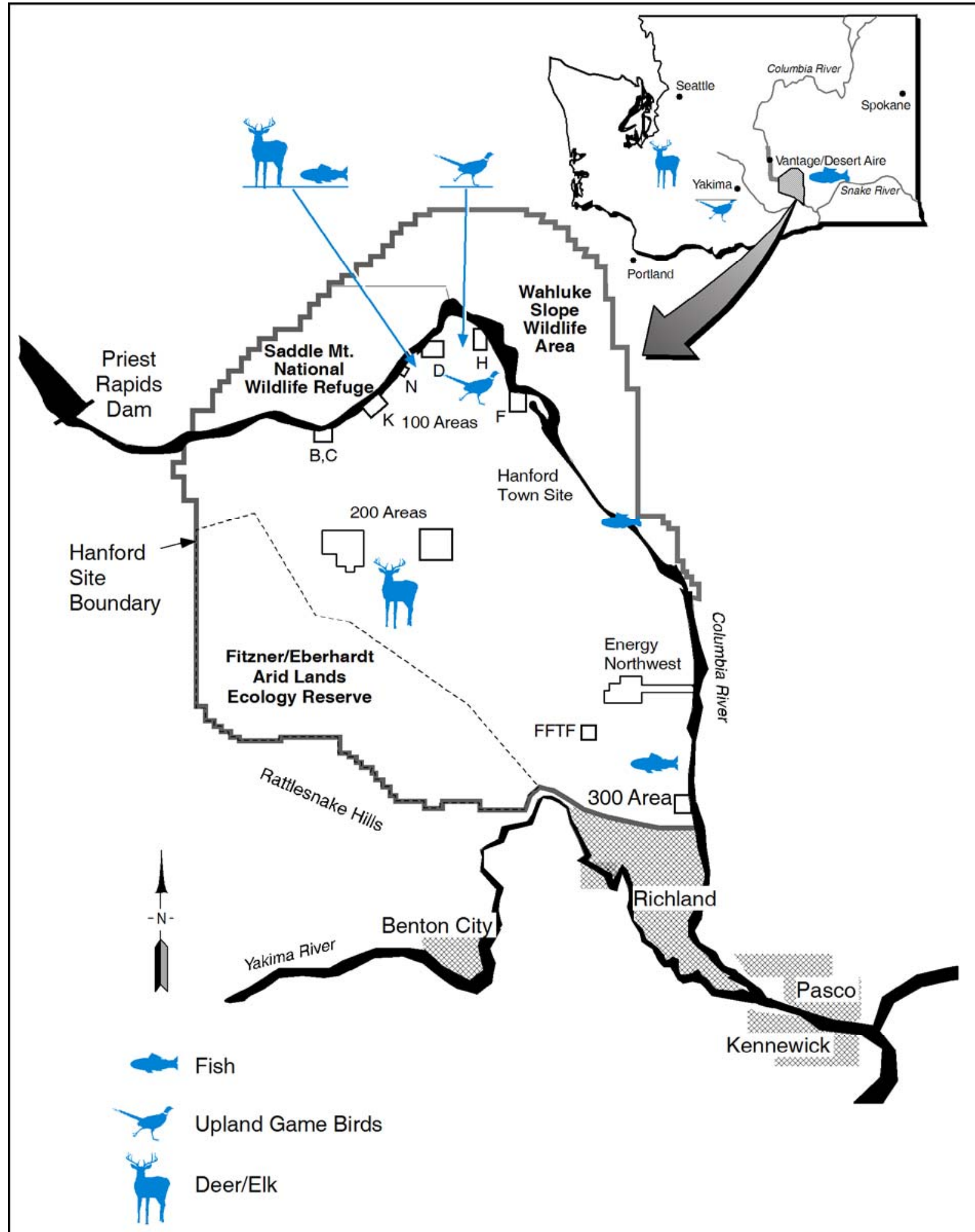

Not to Scale

2009-12-0005-

Figure 4.2. 2010 Wildlife Sampling Locations 


\subsection{Vegetation}

\begin{tabular}{|c|c|c|c|}
\hline Location & Frequency $^{(\mathrm{a})}$ & Collection Period & Analyses/Agency \\
\hline $100 \mathrm{~K}$ Area & 3 to 5 yrs & June-Sept & Gamma Scan, ${ }^{90} \mathrm{Sr}, \mathrm{U}, \mathrm{Pu} / \mathrm{DOH}^{(\mathrm{b})}$ \\
\hline NE of $100 \mathrm{~N}$ Area & 3 to $5 \mathrm{yrs}$ & June-Sept & Gamma Scan, ${ }^{90} \mathrm{Sr}, \mathrm{U}, \mathrm{Pu} / \mathrm{DOH}^{(\mathrm{b})}$ \\
\hline $\mathrm{E}$ of $100 \mathrm{~N}$ Area & 3 to 5 yrs & June-Sept & Gamma Scan, ${ }^{90} \mathrm{Sr}, \mathrm{U}, \mathrm{Pu}$ \\
\hline 100N Spring Shoreline & 3 to 5 yrs & June-Sept & Gamma Scan, ${ }^{90} \mathrm{Sr}, \mathrm{U}, \mathrm{Pu}$ \\
\hline E of 200 W Gate & 3 to 5 yrs & June-Sept & Gamma Scan, ${ }^{90} \mathrm{Sr}, \mathrm{U}, \mathrm{Pu}$ \\
\hline 300 Area Shoreline & 3 to 5 yrs & June-Sept & Gamma Scan, ${ }^{90} \mathrm{Sr}, \mathrm{U}, \mathrm{Pu} / \mathrm{DOH}^{(\mathrm{b})}$ \\
\hline Hanford Townsite & 3 to 5 yrs & June-Sept & Gamma Scan, ${ }^{90} \mathrm{Sr}, \mathrm{U}, \mathrm{Pu}$ \\
\hline Hanford Twnsite HRM28 & 3 to $5 \mathrm{yrs}$ & June-Sept & Gamma Scan, ${ }^{90} \mathrm{Sr}, \mathrm{U}, \mathrm{Pu} / \mathrm{DOH}^{(\mathrm{b})}$ \\
\hline Ringold Area & 3 to 5 yrs & June-Sept & Gamma Scan, ${ }^{90} \mathrm{Sr}, \mathrm{U}, \mathrm{Pu}$ \\
\hline Sagemoor Farm & 3 to 5 yrs & June-Sept & Gamma Scan, ${ }^{90} \mathrm{Sr}, \mathrm{U}, \mathrm{Pu}$ \\
\hline Byers Landing & 3 to 5 yrs & June-Sept & Gamma Scan, ${ }^{90} \mathrm{Sr}, \mathrm{U}, \mathrm{Pu}$ \\
\hline Riverview-Harris & 3 to 5 yrs & June-Sept & Gamma Scan, ${ }^{90} \mathrm{Sr}, \mathrm{U}, \mathrm{Pu}$ \\
\hline Sunnyside & 3 to 5 yrs & June-Sept & Gamma Scan, ${ }^{90} \mathrm{Sr}, \mathrm{U}, \mathrm{Pu}$ \\
\hline Toppenish & 3 to 5 yrs & June-Sept & Gamma Scan, ${ }^{90} \mathrm{Sr}, \mathrm{U}, \mathrm{Pu}$ \\
\hline George & 3 to 5 yrs & June-Sept & Gamma Scan, ${ }^{90} \mathrm{Sr}, \mathrm{U}, \mathrm{Pu} / \mathrm{DOH}^{(\mathrm{b})}$ \\
\hline Othello & 3 to 5 yrs & June-Sept & Gamma Scan, ${ }^{90} \mathrm{Sr}, \mathrm{U}, \mathrm{Pu} / \mathrm{DOH}^{(\mathrm{b})}$ \\
\hline Wanapum & 3 to 5 yrs & June-Sept & Gamma Scan, ${ }^{90} \mathrm{Sr}, \mathrm{U}, \mathrm{Pu} / \mathrm{DOH}^{(\mathrm{b})}$ \\
\hline
\end{tabular}

(a) Samples are collected once every 3 to 5 years and were collected in 2008. Next collection will occur between 2011 and 2013.

(b) Additional sample provided to the $\mathrm{DOH}$. 


\subsection{Sediment}

\subsection{Columbia River}

\begin{tabular}{|c|c|c|}
\hline Location $^{(a)}$ & Frequency & Analyses/Agency \\
\hline \multicolumn{3}{|l|}{ McNary Dam } \\
\hline McNary-OR. Side Near Dam & A & Gamma Scan, ${ }^{90} \mathrm{Sr}, \mathrm{U}, \mathrm{Pu}, \mathrm{ICP}-\mathrm{MS}, \mathrm{Hg}-\mathrm{CVAA}, \mathrm{TOC} / \mathrm{DOH}^{(\mathrm{b})}$ \\
\hline McNary-Wash. Side Near Dam & A & Gamma Scan, ${ }^{90} \mathrm{Sr}, \mathrm{U}, \mathrm{Pu}, \mathrm{ICP}-\mathrm{MS}, \mathrm{Hg-CVAA}, \mathrm{TOC} / \mathrm{DOH}^{(\mathrm{b})}$ \\
\hline \multicolumn{3}{|l|}{ Priest Rapids Dam (PRD) } \\
\hline PRD-Grant Side Near Dam & A & Gamma Scan, ${ }^{90} \mathrm{Sr}, \mathrm{U}, \mathrm{Pu}, \mathrm{ICP}-\mathrm{MS}, \mathrm{Hg}-\mathrm{CVAA}, \mathrm{TOC} / \mathrm{DOH}^{(\mathrm{b})}$ \\
\hline PRD-Yakima Side Near Dam & A & Gamma Scan, ${ }^{90} \mathrm{Sr}, \mathrm{U}, \mathrm{Pu}, \mathrm{ICP}-\mathrm{MS}, \mathrm{Hg-CVAA}, \mathrm{TOC} / \mathrm{DOH}^{(\mathrm{b})}$ \\
\hline White Bluffs Slough & A & Gamma Scan, ${ }^{90} \mathrm{Sr}, \mathrm{U}, \mathrm{Pu}$, ICP-MS, Hg-CVAA, TOC/DOH ${ }^{(\mathrm{b})}$ \\
\hline 100 F Slough & A & Gamma Scan, ${ }^{90} \mathrm{Sr}, \mathrm{U}, \mathrm{Pu}, \mathrm{ICP}-\mathrm{MS}, \mathrm{Hg}-\mathrm{CVAA}$, TOC \\
\hline Hanford Slough & A & Gamma Scan, ${ }^{90} \mathrm{Sr}, \mathrm{U}, \mathrm{Pu}, \mathrm{ICP}-\mathrm{MS}, \mathrm{Hg}-\mathrm{CVAA}$, TOC \\
\hline Richland & A & Gamma Scan, ${ }^{90}$ Sr, U, Pu, ICP-MS, Hg-CVAA, TOC \\
\hline $\begin{array}{l}\text { (a) Refer to Figure 6.1, "2010 Se } \\
\text { (b) Additional sample provided t }\end{array}$ & $\begin{array}{l}\text { mpling L } \\
\text { f. }\end{array}$ & \\
\hline
\end{tabular}

\subsection{River Shoreline Springs}

\begin{tabular}{|c|c|c|c|}
\hline Location $^{(a)}$ & $\mathrm{HRM}^{(\mathrm{b})}$ & Frequency & Analyses/Agency \\
\hline 100-B Spring 38-3 & 3.8 & A & Gamma Scan, ${ }^{90} \mathrm{Sr}, \mathrm{U}$, ICP-MS, Hg-CVAA \\
\hline 100-K Spring 63-1 & 6.3 & A & Gamma Scan, ${ }^{90} \mathrm{Sr}, \mathrm{U}, \mathrm{ICP}-\mathrm{MS}, \mathrm{Hg}-\mathrm{CVAA} / \mathrm{DOH}^{(\mathrm{c})}$ \\
\hline 100-H Spring 145-1 & 14.4 & A & Gamma Scan, ${ }^{90} \mathrm{Sr}, \mathrm{U}, \mathrm{ICP}-\mathrm{MS}$, Hg-CVAA \\
\hline 100-F Spring 207-1 & 21.3 & A & Gamma Scan, ${ }^{90} \mathrm{Sr}, \mathrm{U}$, ICP-MS, Hg-CVAA \\
\hline Hanford Spr UR 28-2 ${ }^{(\mathrm{d})}$ & 27.8 & A & Gamma Scan, ${ }^{90} \mathrm{Sr}, \mathrm{U}, \mathrm{ICP}-\mathrm{MS}, \mathrm{Hg}-\mathrm{CVAA} / \mathrm{DOH}^{(\mathrm{c})}$ \\
\hline Hanford Spr DR 28-2 $2^{(\mathrm{e})}$ & 28.3 & A & Gamma Scan, ${ }^{90} \mathrm{Sr}, \mathrm{U}, \mathrm{ICP}-\mathrm{MS}$, Hg-CVAA/DOH ${ }^{(\mathrm{c})}$ \\
\hline 300 Area Spring 41-9 & 41.9 & A & Gamma Scan, U \\
\hline 300 Area Spring 42-2 & 42.1 & A & Gamma Scan, ${ }^{90} \mathrm{Sr}, \mathrm{U}$, ICP-MS, Hg-CVAA/DOH ${ }^{(\mathrm{c})}$ \\
\hline 300 Area Spr DR 42-2 ${ }^{(\mathrm{e})}$ & 42.4 & A & Gamma Scan, ${ }^{90} \mathrm{Sr}, \mathrm{U}, \mathrm{ICP}-\mathrm{MS}, \mathrm{Hg}-\mathrm{CVAA} / \mathrm{DOH}^{(\mathrm{c})}$ \\
\hline 300 Area Spring 42-7 & 42.7 & A & Gamma Scan, U \\
\hline Richland Spr(SRL 437-1) & 43.7 & A & Gamma Scan, U \\
\hline
\end{tabular}

(a) Refer to Figure 6.1, “2010 Sediment Sampling Locations.”

(b) HRMs are a series of signposts along the Hanford Site shoreline of the Columbia River that are roughly $1.6 \mathrm{~km}$ (1 mi) apart. The Vernita Bridge is HRM \#0 and Ferry Street in Richland is HRM \#46. Samples collected between HRMs are assigned a decimal.

(c) Additional sample provided to the $\mathrm{DOH}$.

(d) UR = Upriver from noted location.

(e) DR = Downriver from noted location.

\subsection{Onsite Pond}

\begin{tabular}{lcc}
\hline \multicolumn{2}{c}{ Location $^{\text {(a) }}$} & Analyses/Agency \\
\hline West Lake & SA (Feb \& June) $\quad$ Gamma Scan, ${ }^{90}$ Sr, U, ${ }^{99}$ Tc, Alpha, Beta \\
\hline (a) Refer to Figure 6.1, "2010 Sediment Sampling Locations.” \\
\hline
\end{tabular}




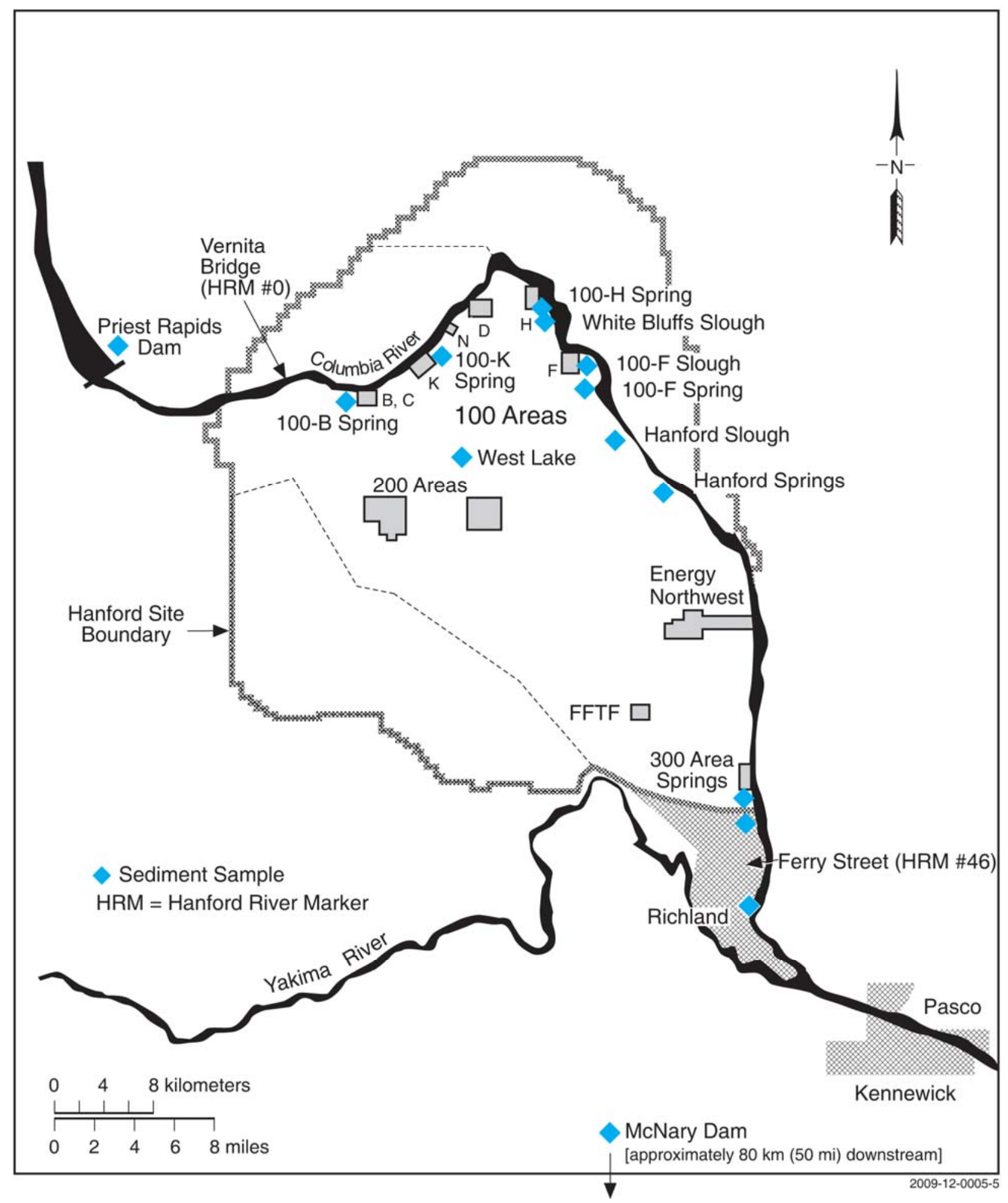

Figure 6.1. 2010 Sediment Sampling Locations 


\section{Distribution}

No. of

Copies

\section{OFFSITE (29)}

2 L. Albin

Division of Radiation Protection

Washington State Department of Health

P.O. Box 47827

Olympia, WA 98504-7827

L. Albright

U.S. Fish and Wildlife Service

64 Maple Street

Burbank, WA 99323

C. Andrade

National Oceanic and Atmospheric

Administration

DARC 7600

Sandpoint Way NE

Seattle, WA 98115

G. Bohnee

Nez Perce Tribe

P.O. Box 365

Lapwai, ID 83540

R. Brunoe, GW Natural Resource

Confederated Tribes of Warm Springs

Reservation

P.O. Box 1299

Warm Springs, OR 97761

L. Buck

Wanapum

P.O. Box 878

Ephrata, WA 98823

R. Buck, Jr.

Wanapum

P.O. Box 878

Ephrata, WA 98823

L. Buelow

U.S. Environmental Protection Agency, 309 Bradley, Suite 115, MS B1-46

Richland, WA 99352
No. of

Copies

L. Gadbois

U.S. Environmental Protection Agency

309 Bradley, Suite 115, MS B1-46

Richland, WA 99352

L. Goldstein

Washington State Department of Ecology

P.O. Box 47600

Olympia, WA 98504

B. Harper

Confederated Tribes of the Umatilla Indian

Reservation

750 Swift, Suite 14

Richland, WA 99352

S. Harris

Confederated Tribes of the Umatilla Indian

Reservation

P.O. Box 638

Pendleton, OR 97801

G. Hughes

U.S. Fish and Wildlife Service

64 Maple Street

Burbank, WA 99323

R. Jim, Manager

Yakama Nation

2808 Main Street

Union Gap, WA 98903

E. Kramer

Division of Radiation Protection

Washington State Department of Health

P.O. Box 47827

Olympia, WA 98504-7827

D. Landeen

Hanford Natural Resources Trustee

Council

Nez Perce Tribe

P.O. Box 365

Lapwai, ID 83540

Distr. 1 
PNNL-19079

No. of

Copies

D. McBaugh

Division of Radiation Protection

Washington State Department of Health

P.O. Box 47827

Olympia, WA 98504-7827

J. McConnaughey

Yakama Nation

P.O. Box 6066

Kennewick, WA 99336-0066

A. Nomee

Department of Natural Resources

Coeur d'Alene Tribe

P.O. Box 408

Plummer, ID 83851-9703

Office of Environmental Cleanup

Director - Hanford Project Office

$12006^{\text {th }}$ Avenue

Seattle, WA 98101

C. Pleasants

Confederated Tribes of the Colville Indian

Reservation

P.O. Box 150

Nespelem, WA 99155

M. Priddy

Washington State Department of Health

309 Bradley, Suite 201

Richland, WA 99352

P. Rigdon

Yakama Nation

P.O. Box 151

Toppenish, WA 98948

B. Ruben

Washington State Department of Health 309 Bradley, Suite 201

Richland, WA 99352

P. Shaffer

Oregon Department of Energy

625 Marion Street NE

Salem, OR 97301-3737
No. of

Copies

J. Skriletz

Washington Department of Fish and

Wildlife

600 Capitol Way

1111 Washington Street NE

Olympia, WA 98501-1091

T. Southworth

Energy Northwest

P.O. Box 968

Richland, WA 99354

S.P. Van Verst

Division of Radiation Protection

Washington State Department of Health

P.O. Box 47827

Olympia, WA 98504-7827

ONSITE (72)

DOE Pacific Northwest Site Office

J. Erickson K9-42

21 DOE Richland Operations Office

D.A. Brockman

A7-50

N. Ceto

A7-50

R.J. Corey

A5-14

B.L. Foley

A6-38

J.R. Franco

A3-04

J.A. Hansen

A5-11

R.G. Hastings

A5-19

A.R. Hawkins A5-14

J.P. Sands A3-04

K.M. Thompson A6-38

A.C. Tortoso A6-38

G.T. Tyree A7-75

D.C. Ward (5) A5-15

S.R. Weil A5-15

J. Zeisloft A3-04

DOE Public Reading Room (2) H2-53

2 DOE Office of River Protection

L.A. Huffman H6-60

W. Russell H6-60 
No. of

Copies

6 Mission Support Alliance, LLC

J.J. Dorian

H7-28

A.R. Johnson

L.M. Kelly

S.M. McKinney

C.J. Perkins

K.A. Peterson

R3-12

R3-15

H7-28

H7-28

H7-28

3 Washington Closure Hanford, LLC

K.A. Gano
L.C. Hulstrom
R.W. Ovink

H4-23

H4-22

H4-21

39 Pacific Northwest National Laboratory

E.J. Antonio

M. Barnett
K3-54

J2-25
No. of

Copies

L.E. Bisping (20)

K6-75

J.M. Brandenberger

R.L. Dirkes (2)

J.L. Downs

R.E. Durham

B.G. Fritz

K.D. Hand

B.D. Johns

B.K. Lasorsa

E.A. Lepel

R.P. Mueller

B.E. Opitz

G.W. Patton

R.E. Peterson

T.M. Poston

J.A. Stegen

Historical File-T.M. Poston
Sequim

K6-75

K6-85

K6-85

K6-75

K6-85

K6-75

Sequim

P8-01

K6-85

K6-75

K6-75

K6-75

K6-75

K3-66

K6-75 


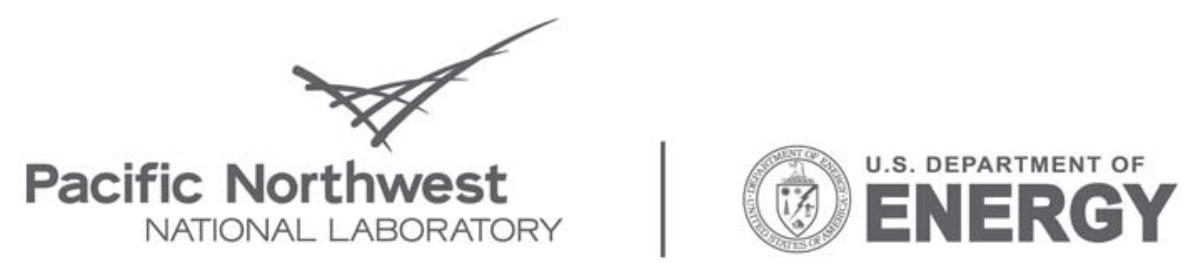

Proudly Operated by Battelle Since 1965

902 Battelle Boulevard

P.O. Box 999

Richland, WA 99352

1-888-375-PNNL (7665)

www.pnl.gov 